\title{
Movilidad residencial en tiempos de crisis. El caso de la Región Metropolitana de Barcelona
}

\author{
Arlinda García Coll \\ Departament de Geografia \\ Universitat de Barcelona \\ arlindagarcia@ub.edu \\ Cristina López Villanueva \\ Departament de Sociologia \\ Universitat de Barcelona \\ clopez@ub.edu \\ Isabel Pujadas Rúbies \\ Departament de Geografia \\ Universitat de Barcelona \\ ipujadas@ub.edu
}

\begin{abstract}
Movilidad residencial en tiempos de crisis. El caso de la Región Metropolitana de Barcelona (Resumen)
\end{abstract}

Este artículo profundiza en el efecto de la crisis actual en la movilidad residencial a partir del estudio de la migración intrametropolitana de la Región Metropolitana de Barcelona. A pesar de una aparente estabilidad en la movilidad residencial tras la irrupción de la crisis, el análisis en profundidad evidencia que ésta reduce de forma sensible la movilidad de los extranjeros, la del grupo 25-34 años y de los mayores de 55 años, así como las migraciones hacia municipios caracterizados por un urbanismo disperso. Por contra, causa un incremento de la migración con destino a áreas compactas. La conclusión es que nos encontramos en un momento de transición, en el que los municipios con predominio de residencia de baja densidad siguen presentando ganancias migratorias netas a pesar de la disminución progresiva de su inmigración. En cambio, los municipios compactos siguen siendo todavía expulsores netos de población, aunque aumentan su capacidad de atracción. En el caso de la Región Metropolitana de Barcelona, la crisis actúa frenando el proceso de dispersión residencial, a la vez que revaloriza las áreas compactas que ganan ahora atractivo como lugar de destino de las migraciones intrametropolitanas.

Palabras clave: Movilidad residencial, Región Metropolitana de Barcelona, estrategias residenciales, urbanismo disperso, migración intrametropolitana. 


\section{Residential mobility during the economic crisis. The Barcelona Metropolitan Region's case (Abstract)}

This article focuses on the effect of the crisis on residential mobility from the study of intrametropolitan migration of the Metropolitan Region of Barcelona. Despite the apparent stability of residential mobility after the outbreak of the crisis, in-depth analysis shows that the crisis reduces significantly the mobility of foreigners, the group 25-34 years and those older than 55 years, as well as movements to sprawl municipalities. In contrast, there is an increase in immigration to compact areas. The conclusion is that we are in a time of transition in which the sprawl areas still show net migration gains, despite the progressive decrease in immigration. Instead, compact municipalities have still negative net migration rates, although they are increasing their immigration. In the case of the RMB, the crisis works by slowing the process of residential dispersion, while enhancing values in compact areas that earn attractive as a destination for intra-metropolitan migration.

Key words: Residential mobility, Barcelona Metropolitan Region, residential strategies, urban sprawl, intrametropolitan migration.

Desde el año 2007, España se ha visto inmersa en una crisis económica de gran calado, la cual ha ido transformando la realidad de la sociedad española desde el momento de su irrupción hasta el presente'. A nivel demográfico, la literatura científica más reciente ha abordado un amplio abanico de efectos generados por la crisis $^{2}$, de forma paralela a la aparición de nuevas estadísticas que permiten constatar de forma más diáfana la influencia de la misma en los distintos campos relacionados con la población. Se trata, pues, de un escenario en construcción, donde las pautas vigentes hasta el momento empiezan a diluirse y, poco a poco, empieza a definirse un nuevo contexto sociodemográfico.

En este contexto, el análisis del papel de la crisis en la movilidad residencial despierta, si cabe, todavía mayor interés, debido a tres razones fundamentales. En primer lugar, porque en la etapa inmediatamente anterior la movilidad residencial en España había experimentado un crecimiento espectacular ${ }^{3}$, en especial entre 2002 y 2006 y, por tanto, queda pendiente evaluar la excepcionalidad o permanencia de dicho comportamiento. Este balance debe incidir ya no sólo en la intensidad adquirida por la movilidad, sino también por la ampliación de los municipios involucrados en el intercambio de residentes a escala metropolitana y por la importancia adquirida por las áreas de baja densidad, que en estos momentos se convierten en polos de atractivo inmigratorio. En segundo lugar, debido a que la evaluación del impacto de la crisis en la movilidad aporta evidencias empíricas a un viejo debate teórico que intenta establecer relaciones entre intensidad migratoria de la población y ciclos económicos,

\footnotetext{
1 Este trabajo ha sido realizado en el marco de tres proyectos de investigación: "Estrategias residenciales y modelos urbanos en la Región Metropolitana de Barcelona" (CSO 2010-22117-C02-02), "Cambio social y procesos de transformación urbana en un contexto de crisis en las periferias urbanas de las grandes áreas metropolitanas de España. El caso de la RMB” (CSO2013-48075-C2-1-R) y "Desigualdad social, polarización territorial y formación de espacios vulnerables en las grandes áreas metropolitanas españolas" (CSO2015-65219-C2-1-R), financiados por el actual Ministerio de Economía y Competitividad (MINECO/FEDER, UE).

2 Domínguez Mujica, 2013; Colectivo IOE, 2012 y Reher et al., 2011.

${ }^{3}$ Susino y Duque, 2013; Gil-Alonso et al., 2016 y García Coll, 2009.
} 
es decir, cómo las distintas coyunturas económicas actúan favoreciendo o frenando los flujos migratorios ${ }^{4}$ o simplemente transformándolos. Finalmente, el estudio de los cambios en la movilidad residencial entronca directamente con la discusión teórica sobre la desigual incidencia de la crisis en los distintos colectivos. De esta manera, las diferencias según edad -como sucede en el caso de los jóvenes-, nacionalidad -en especial en lo que se refiere a población autóctona o extranjera- o nivel socioeconómico se convierten en elementos fundamentales para aproximarse a un análisis afinado de la realidad ${ }^{5}$.

\section{Un nuevo escenario en construcción. El efecto de la crisis actual en el cambio demográfico reciente}

Este artículo realiza una aportación a estos tres debates señalados a partir del análisis de la movilidad residencial en la Región Metropolitana de Barcelona (RMB), ámbito que cuenta en 2013 con más de 5 millones de habitantes y que registra, en los últimos años, cifras superiores a las 140.000 migraciones intrametropolitanas anuales.

Los objetivos de la presente investigación son, en definitiva, los siguientes. En primer lugar, contrastar los comportamientos registrados por la movilidad residencial en la RMB en el momento de crisis económica (período 2008-2012), frente a la etapa anterior marcada por un acentuado crecimiento (2001-2007), de manera que sea posible extraer conclusiones sobre cómo la crisis está modificando los intercambios migratorios intrametropolitanos. En segundo lugar, el análisis se plantea comparar las trayectorias de los municipios compactos respecto a aquellos con mayor protagonismo del modelo de baja densidad que son, precisamente, los que experimentaron un crecimiento más acusado en la etapa inmediatamente anterior, coincidiendo con el boom de la movilidad residencial. Esta perspectiva requiere la clasificación de los municipios de la RMB de acuerdo con el grado de compacidad de los mismos.

Finalmente, el tercer objetivo consiste en el análisis diferenciado según la edad de los migrantes y según se trate de españoles o extranjeros. Los resultados de este análisis permiten la obtención de conclusiones sobre la incidencia desigual de la crisis tanto desde el punto de vista de las características territoriales (compacto/disperso) como de las personas (adultos-jóvenes, mayores o extranjeros).

El estudio de los cambios en la movilidad interna la RMB es un tema de gran relevancia en la configuración actual de las regiones metropolitanas, ya que ésta ha venido actuando como el principal motor del crecimiento demográfico local, contribuyendo activamente no sólo a la redistribución de la población de la RMB, sino también a acentuar los procesos de cambio estructural y segregación social ${ }^{6}$.

\footnotetext{
${ }^{4}$ Greenwood, 1993; Ródenas, 1994.

5 como se refleja en trabajos del Colectivo IOE (2009), Aja et al. (2013) o Domingo y Recaño (2009).

${ }^{6}$ Susino y Duque, 2013; Gil-Alonso et al, 2013; Susino, 2010; Pujadas y García Coll, 2007 y García Almirall et al. 2008.
} 


\section{Fuentes y metodología: elementos para un análisis territorial de la movilidad residencial en España.}

El estudio de las transformaciones urbanas en los últimos decenios se puede llevar a cabo desde diferentes perspectivas y escalas de análisis: coronas metropolitanas, tamaño demográfico del municipio, tipos de municipio, distancia respecto a la ciudad central, entre otras. En el caso de la RMB, uno de los aspectos que mejor ayuda a entender la lógica de las transformaciones recientes la proporciona la expansión del urbanismo disperso, que distingue entre la ciudad compacta y un modelo de ocupación urbana de baja densidad. Es por este motivo que esta investigación emplea una tipología que clasifica los municipios de la RMB según su grado de compacidad o dispersión, tipología que ya se había aplicado, con variables similares en un estudio anterior para la propia $\mathrm{RMB}^{7}$.

El primer paso para la caracterización del urbanismo disperso en la RMB ha sido, por tanto, la clasificación de sus municipios según el modelo predominante de ocupación urbana: ciudad compacta y de alta densidad o bien urbanismo disperso o de baja densidad. La clasificación se realiza por medio de un análisis de conglomerados a partir de cuatro variables que recogen aspectos claves para identificar la importancia del urbanismo disperso, fieles a las consideraciones teóricas establecidas por López de Lucio o Muñiz ${ }^{8}$. Se incorpora: el porcentaje de superficie residencial dedicada a viviendas unifamiliares y en urbanizaciones en 2001, la densidad neta municipal de suelo residencial en 2001, la proporción de casas unifamiliares construidas entre 19962001 y, finalmente, la tasa de construcción de nuevas viviendas en el período 19962001. Estas variables se refieren, de acuerdo con la propuesta de periodificación de la evolución de la RMB que señala Alabart ${ }^{9}$, a un momento intermedio del fenómeno de dispersión urbana, previo a la etapa del crecimiento más intenso y tras un período de conformación del modelo.

\section{Cuadro 1}

Clasificación de los municipios de la RMB según compacidad/dispersión

\begin{tabular}{|c|c|c|c|c|c|c|}
\hline & \multicolumn{2}{|c|}{ Municipios } & \multirow{2}{*}{$\begin{array}{c}\text { Densidad neta } \\
\text { (hab./km2) } \\
2001\end{array}$} & \multirow{2}{*}{$\begin{array}{l}\text { \% Superf. Residencial } \\
\text { en viviendas unifam. } \\
2001\end{array}$} & \multirow{2}{*}{$\begin{array}{c}\text { \% Viviendas unifam. } \\
\text { sobre total construidas } \\
1996-2001\end{array}$} & \multirow{2}{*}{$\begin{array}{c}\text { Tasa construcción } \\
\text { viviendas }(\% \text { ) } \\
1996-2001\end{array}$} \\
\hline & $\mathrm{n}$ & $\%$ & & & & \\
\hline Compacidad muy alta & 7 & 4,3 & 415,3 & 7,1 & 12,1 & 2,5 \\
\hline Compacidad alta & 17 & 10,4 & 242,9 & 27,6 & 15,2 & 8,9 \\
\hline Mixtos & 33 & 20,1 & 106,9 & 66,3 & 25,2 & 13,2 \\
\hline Dispersión media & 31 & 18,9 & 47,7 & 84,4 & 49 & 15,2 \\
\hline Dispersón máxima & 53 & 32,3 & 25,9 & 87,7 & 78,3 & 10,4 \\
\hline$<1.000$ habitantes & 23 & 14,0 & 31,7 & 87,9 & 79,1 & 14,7 \\
\hline Total & 164 & 100,0 & 130 & & & 6,15 \\
\hline
\end{tabular}

Fuente: Elaboración propia a partir de INE: Censo de Población y Vivienda, 2001; INE: Nomenclator, 2001, Certificados final de obra del Colegio de Aparejadores de Barcelona y Mapa de usos del suelo de la RMB del Servicio de Estudios Territoriales del Área Metropolitana de Barcelona.

La agrupación de municipios resulta de la estandarización de las variables, empleando el método de Ward sobre la distancia euclídea al cuadrado. El resultado obtenido ha agrupado 5 tipos de municipios que responden a características distintas en cuanto a contenido. Al margen de la tipología se han dejado los municipios con una población

\footnotetext{
${ }^{7}$ Pujadas et al., 2007.

${ }^{8}$ López de Lucio, 1998; Muñiz, 2006.

${ }^{9}$ A. Alabart, 2007.
} 
inferior a los 1.000 habitantes, puesto que el reducido tamaño de los valores de las variables empleadas en la clasificación daba lugar a indicadores extremos y de gran aleatoriedad.

El resultado es la agrupación de los 164 municipios de la RMB en 5 categorías que responden a las características que muestra el cuadro 1 y la distribución que ilustra la figura 1.

Figura 1

Tipología municipal según predominio urbanismo disperso/compacto

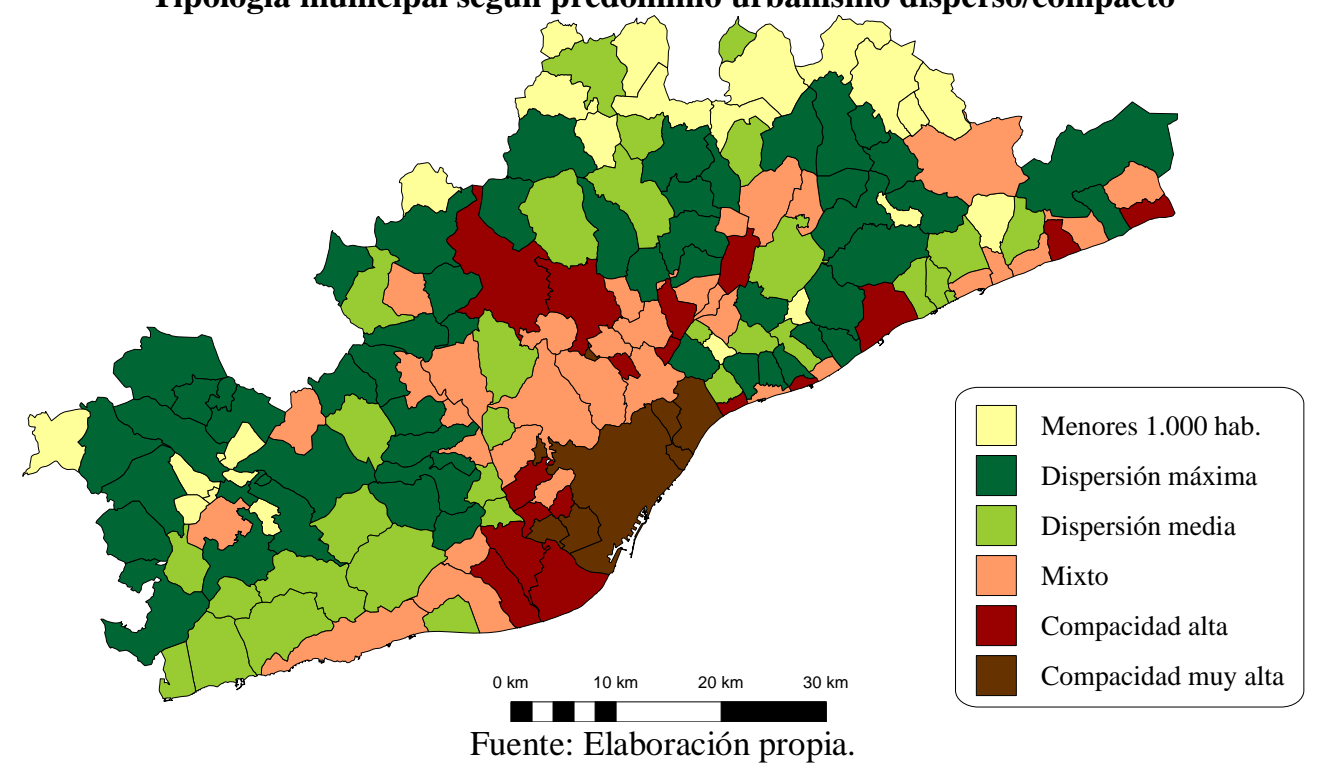

En primer lugar se distinguen los municipios de compacidad muy alta. Se trata de municipios caracterizados por una densidad neta media muy elevada (415 hab. $/ \mathrm{km}^{2}$, prácticamente el doble que la de la categoría siguiente) y un bajo porcentaje de superficie residencial destinada a viviendas unifamiliares $(7 \%)$. Esta categoría reúne a un total de 7 municipios entre los que se encuentra la ciudad de Barcelona y los municipios de su inmediata área de influencia, como son Sant Adrià del Besòs, Badalona, Santa Coloma de Gramenet, L'Hospitalet de Llobregat, Cornellà de Llobregat, además de Badia del Vallès.

En segundo lugar se encuentra el grupo de compacidad alta. En esta categoría se incluyen municipios con una densidad neta elevada $\left(243 \mathrm{hab} . / \mathrm{km}^{2}\right)$ y un porcentaje superior de superficie al de la categoría anterior dedicada a la vivienda unifamiliar (28\%). Este grupo incluye el resto de municipios de la antigua área metropolitana de Barcelona, es decir, el ámbito de crecimiento de la ciudad de Barcelona en su etapa de expansión durante la fase del desarrollismo, incluyendo municipios muy cercanos a la capital como son El Prat, Esplugues, Sant Boi, Sant Feliu de Llobregat, Viladecans y Sant Joan Despí. Además incorpora las grandes ciudades de la RMB, como Sabadell, Terrassa, Mataró y Granollers y algunos de los municipios más poblados del área del Vallès (La Llagosta, Mollet, Ripollet) y del Maresme (Calella, Malgrat y Premià de Mar).

En tercer lugar aparecen los municipios mixtos, conjunto formado por 33 municipios que combinan una elevada densidad $\left(106,9 \mathrm{hab} . / \mathrm{km}^{2}\right)$, un alto porcentaje de superficie residencial en viviendas unifamiliares $(66,3 \%)$ y una intensa tasa de construcción 
$(13,2 \%$ ). Se trata de municipios situados, algunos, en una zona de transición entre el compacto y disperso, e incluye municipios costero-residenciales (como Sitges, Vilassar de Mar, Sant Pol de Mar, Canet de Mar) o interiores residenciales (como, por ejemplo, Sant Cugat del Vallès, Cerdanyola del Vallès, Sant Just Desvern o Cardedeu).

En cuarto lugar se distinguen los municipios de dispersión media. Esta categoría agrupa 31 municipios repartidos por toda la Región, sin una concentración específica, más bien situados a cierta proximidad de los municipios de mayor tamaño, como Sant Pere de Ribes (contiguo a Sitges), Sant Quirze del Vallès y Castellar del Vallès (vecinos a Sabadell), entre otros.

Finalmente, se halla el grupo de los municipios de dispersión máxima. Este grupo representa la máxima expresión del sprawl en la RMB. Se concentra principalmente en zonas boscosas de montaña media o bien en zonas litorales que tienen una presencia importante de urbanizaciones y segundas residencias. Este es el caso de la mayoría de los municipios del Ordal-Garraf, próximos al eje del Llobregat, de los municipios de la coordillera prelitoral del Maresme y de algunos de la depresión prelitoral del Vallès.

A partir de los resultados de esta categorización es posible la reorganización de la información proporcionada por las estadísticas estándar en función del tipo de municipio y contrastar los comportamientos básicos según grado de compacidad. Por un lado, los datos del Padrón Municipal de Habitantes permiten evaluar las diferencias en el crecimiento demográfico. Por otra parte, la utilización de los microdatos de la Estadística de Variaciones Residenciales (EVR) ofrece información anual sobre el número de inmigrantes y emigrantes intrametropolitanos recibidos por cada uno de los municipios y brinda una excelente imagen de la movilidad residencial intrametropolitana y de los cambios experimentados durante el período 1996-2012. Esta fuente no está exenta de algunos problemas de registro, entre los que destaca el descenso sistemático de cambios de residencia coincidiendo con los años padronales y censales (1996 y 2001).

Otra de las limitaciones de la fuente es el caso de las altas por omisión que hasta el 2004 eran incorporadas al Padrón sin registrarse como auténticas migraciones en las EVR. La repercusión de esta práctica es muy irregular pero es especialmente importante en los años de fuerte llegada de inmigración extranjera, máxime durante el período 2001-03. A partir del año 2004, las altas por omisión se añaden a las $E V R$ como un cambio más de municipio de residencia, lo que corrige el subregistro padecido hasta ese momento.

Finalmente, cabe recordar que en 2006 se aplica por primera vez el Decreto que exige la renovación de la inscripción padronal cada dos años de los residentes extranjeros no comunitarios sin permiso de residencia permanente. La ausencia de este trámite significa la baja automática de la persona del Padrón por caducidad. La aplicación de este Decreto tiene un impacto directo tanto en el cómputo de los stocks como de los flujos de población extranjera. 


\section{Transformaciones recientes en la evolución de la población de los municipios de la RMB. La desigual trayectoria de los municipios compactos y dispersos.}

La RMB cuenta, a fecha 1 de enero de 2012, con 5.051.502 habitantes (cuadro 2). Más allá de la cifra total de habitantes que registra, el interés de la evolución de la población de la Región redunda en las profundas transformaciones experimentadas en el transcurso de las dos últimas décadas (figura 2).

Cuadro 2

Población según tipo de municipio (Totales y \%)

\begin{tabular}{|l|r|r|r|r|r|r|}
\hline \multirow{2}{*}{ Tipología } & \multicolumn{3}{|c|}{ Población total } & \multicolumn{3}{c|}{ Distribución (\%) } \\
\cline { 2 - 7 } & \multicolumn{1}{|c|}{$\mathbf{1 9 9 6}$} & \multicolumn{1}{c|}{$\mathbf{2 0 0 1}$} & $\mathbf{2 0 1 2}$ & $\mathbf{1 9 9 6}$ & $\mathbf{2 0 0 1}$ & $\mathbf{2 0 1 2}$ \\
\hline Compacidad muy alta & 2.230 .926 & 2.201 .479 & 2.355 .073 & 52,76 & 50,14 & 46,62 \\
Compacidad alta & 945.087 & 986.719 & 1.121 .576 & 22,35 & 22,47 & 22,20 \\
Mixto & 536.133 & 598.136 & 762.287 & 12,68 & 13,62 & 15,09 \\
Dispersión media & 311.397 & 360.525 & 472.928 & 7,37 & 8,21 & 9,36 \\
Dispersión máxima & 194.298 & 232.096 & 323.172 & 4,60 & 5,29 & 6,40 \\
\hline$<1.000$ & 10.207 & 11.458 & 16.466 & 0,24 & 0,26 & 0,33 \\
\hline Total RMB & $\mathbf{4 . 2 2 8 . 0 4 8}$ & $\mathbf{4 . 3 9 0 . 4 1 3}$ & $\mathbf{5 . 0 5 1 . 5 0 2}$ & 100,00 & 100,00 & 100,00 \\
\hline \% extranjeros & 1,6 & 3,8 & 14,4 & & & \\
\hline
\end{tabular}

Fuente: Elaboración propia a partir del INE, Padrón Municipal de Habitantes, 1996 y Padrón Continuo 1998-2012.

Así, su análisis permite distinguir tres etapas contrastadas en el crecimiento de la población. Por un lado, el período 1996-2000, donde el comportamiento predominante es el aumento de las tasas, con un promedio para el período de $0,8 \%$. Esta conducta contrasta con las intensas tasas presentadas entre 2001 y 2007, etapa con un crecimiento medio de 1,7\%. Finalmente, en el período 2008-2012, el crecimiento de la población disminuye de forma notoria, de manera que se alcanzan los mínimos del período, con un discreto $0,6 \%$. En conjunto, cabe destacar el fuerte ritmo de crecimiento experimentado en 2002 y 2004 , cuando la tasa anual supera el $2 \%$. Como resultado, la población de la RMB se incrementó en más de 600.000 personas entre 2001 y 2012, es decir, más del 15\%.

El principal factor explicativo de este crecimiento cabe buscarlo en el intenso flujo de entrada de población extranjera ${ }^{10}$. No en vano, este colectivo aumenta de 66.328 en 1996 a 726.065 en 2012, es decir, se ha multiplicado en casi 11 veces, pasando de representar el 1,6\% de la población residente en la RMB a ser el 14,4\% de la misma. La tendencia al fuerte crecimiento se aminora en los últimos años: entre 2007 y 2008 la tasa de crecimiento pasa a situarse en torno al $1,5 \%$ y a partir de 2009 se coloca en un modesto $0,3-0,4 \%$, consecuencia directa de la reducción de la inmigración internacional. Esta reducción es un fenómeno generalizado que afecta al conjunto de España, pues según datos del Colectivo Ioé ${ }^{11}$, la inmigración hacia España se divide por la mitad entre 2007 y 2011, es decir, de 915.000 a 408.000 personas.

\footnotetext{
${ }^{10}$ Bayona y Gil, 2012; Bayona y López Gay, 2011.

${ }^{11}$ Colectivo IOÉ, 2012.
} 
Figura 2

Evolución de la tasa de crecimiento anual acumulativa (r\%), 1996-2012
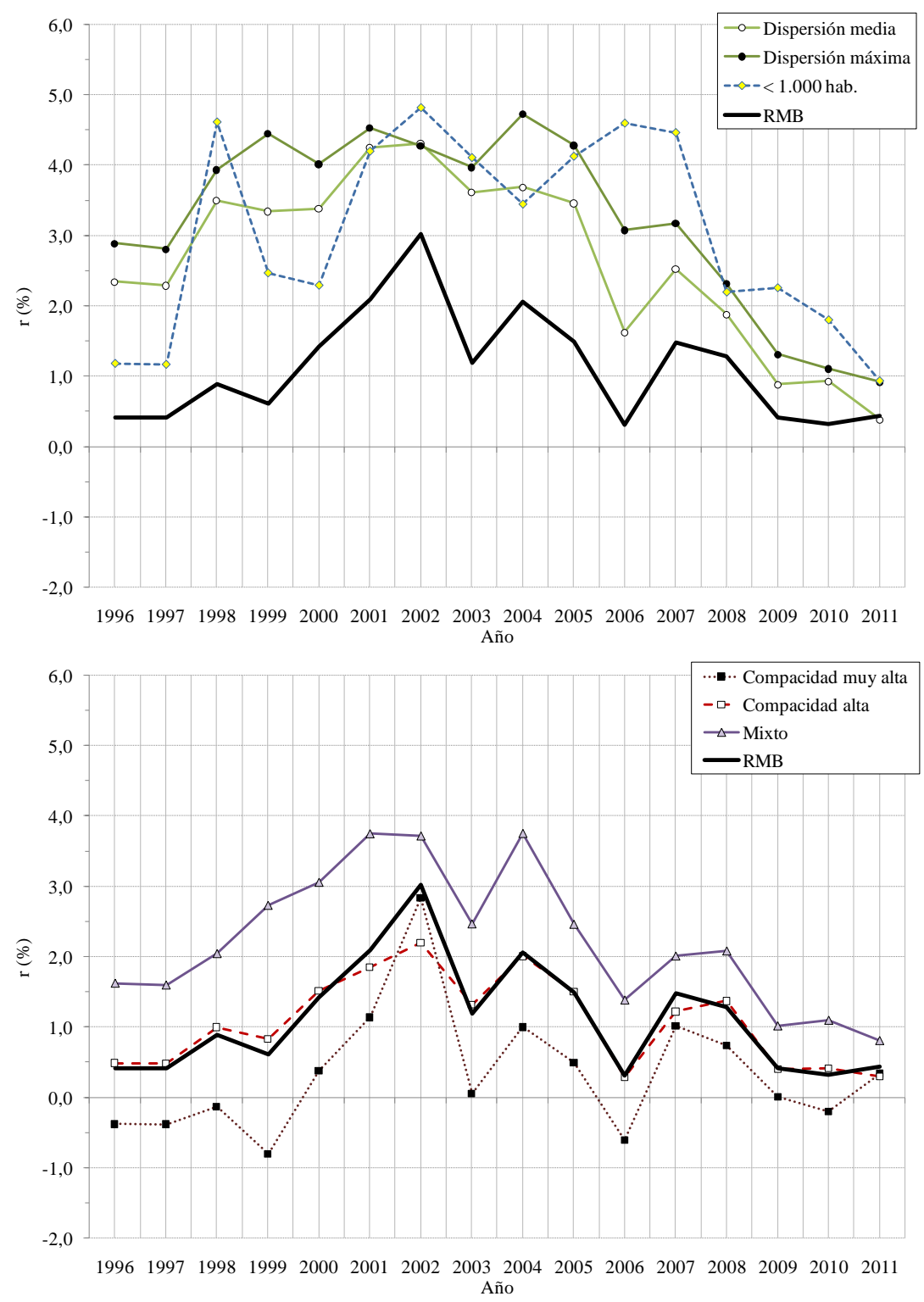

Fuente: Elaboración propia a partir de INE: Padrón Municipal de Habitantes, 1996 y Padrón Continuo, 1998-2012.

A la luz de los datos, es evidente el drástico freno experimentado por el crecimiento de la población metropolitana, si bien ésta se produce de forma más tardía al inicio de la crisis. Según Domínguez Mujica ${ }^{12}$, esta es precisamente una de las características de las tendencias demográficas, las cuales reaccionan a los cambios de coyuntura socieconómica con cierta dilación en el tiempo.

Más allá de la imagen que nos proporcionan los datos agregados, cabe señalar que uno de los elementos más destacados de la evolución demográfica de la RMB en el transcurso del período estudiado es el paulatino proceso de dispersión residencial de su población, tendencia que comienza a finales de los ochenta ${ }^{13}$, pero cuya mayor

\footnotetext{
12 Domínguez Mujica, 2013.

${ }^{13}$ Alabart y López-Villanueva, 2007; Pujadas, 2009 o Muñoz, 2010 y 2011.
} 
intensidad y expansión territorial se concentra entre 1996 y 2007. Este fenómeno se hace evidente cuando se comprueban las tasas de crecimiento de los municipios de dispersión media y dispersión máxima (cuadro 3), con valores que llegan a superar el 4\% anual. Este crecimiento superior a la media metropolitana explica las ganancias registradas por las tipologías de municipios dispersos, que pasan de albergar el 11,9\% de la población al $15,9 \%$ (del $4,5 \%$ al $6,4 \%$ en el caso de los de dispersión máxima y del $7,4 \%$ al 9,4\% en el caso de los de dispersión media).

Cuadro 3

Tasa de crecimiento anual acumulativo $(\mathbf{r} \%)$. 1996-2012.

\begin{tabular}{|l|r|r|r|r|}
\hline \multirow{2}{*}{\multicolumn{1}{c|}{ Tipología }} & \multicolumn{4}{|c|}{ r (\%) } \\
\cline { 2 - 5 } & 1996-2000 & 2001-2007 & 2008-2012 & 1996-2012 \\
\hline Dispersión máxima & 4,0 & 4,0 & 1,4 & 3,3 \\
Dispersión media & 3,3 & 3,4 & 1,0 & 2,7 \\
Mixto & 2,4 & 2,8 & 1,3 & 2,3 \\
Compacidad alta & 0,9 & 1,5 & 0,6 & 1,1 \\
Compacidad muy alta & $-0,3$ & 0,8 & 0,2 & 0,3 \\
\hline$>1.000$ habitantes & 2,6 & 4,2 & 1,8 & 3,1 \\
\hline Total & 0,8 & 1,7 & 0,6 & 1,1 \\
\hline
\end{tabular}

Fuente: Elaboración propia a partir de INE: Padrón Municipal de Habitantes, 1996 y Padrón Continuo 1998-2012.

La cara opuesta se encuentra en la pérdida de población de los municipios más densos y compactos, el clúster de compacidad muy alta, restricción que es continua a lo largo de los años noventa, pues su tasa de crecimiento es negativa hasta el año 2000. Esta circunstancia explica que su peso demográfico no deja de retroceder en el conjunto de la población de la RMB: pasa del 52,8\% al 46,6\% entre 1996-2012 para la suma de los 7 municipios más densos y del 35,7\% al 32,1\% para la ciudad de Barcelona.

A pesar de las nuevas tendencias, es importante señalar que persiste la jerarquía de mayor crecimiento en los municipios dispersos y menor ritmo en los compactos, jerarquía que se mantiene en todo momento, incluso cuando ya la crisis ya está actuando de forma activa. Las tasas anuales de crecimiento en el grupo de dispersión máxima muestran un fuerte descenso en años previos al estallido de la burbuja inmobiliaria. Efectivamente, en 2004 la tasa alcanza el 4,7\%, en el 2007 el 3,2\% y en el 2008 el 2,3\%, y, finalmente, desciende al 0,9\% en el 2011. La crisis se deja notar con unos años de anticipación en los mercados dominados por la vivienda unifamiliar, constatando una saturación de la demanda ante una oferta cada vez más cara. Se observa que los municipios considerados como mixtos en nuestra tipología presentan un crecimiento superior que los de dispersión media desde el estallido de la crisis, mientras que los compactos presentan una recuperación en el último año.

Según Gil et al. ${ }^{14}$, la transformación del crecimiento de la población de la RMB es consecuencia directa de la crisis económica-financiera y del mercado inmobiliario que se hace patente a partir de 2008. La crisis habría frenado parcialmente las dinámicas de reurbanización y de dispersión de baja densidad. Las causas de estos cambios se encuentran, por un lado, en la reducción de la llegada de migración internacional circunstancia que hace disminuir el ritmo de crecimiento de los centros urbanos- y, por

\footnotetext{
${ }^{14}$ Gil-Alonso et al. 2011.
} 
otro, en la dificultad de acceso a nuevas viviendas que frena la movilidad residencial de los españoles y, por tanto, reduce la dispersión residencial. Estos dos factores, en definitiva, explicarían los nuevos comportamientos detectados en el crecimiento de la población de la RMB en los últimos años.

En resumen, la crisis ha representado un brusco freno del crecimiento de la RMB, que hoy por hoy aumenta su población a un ritmo muy moderado. Sin embargo, las transformaciones más pronunciadas se constatan en el ritmo de redistribución interna de la población metropolitana, con comportamientos contrastados entre municipios compactos y dispersos. En este contexto, es fundamental examinar el comportamiento de la movilidad residencial y centrar el análisis detallado en ella, ya que sus pautas serán indicativas de la reformulación en los procesos intrametropolitanos.

\section{Movilidad residencial en tiempos de crisis. Nuevas pautas, nuevos actores, nuevos destinos.}

La dinámica migratoria intrametropolitana de la RMB ha conocido una serie de drásticos cambios en el transcurso de las dos últimas décadas. Por un lado, el número de migraciones anuales va en continuo aumento hasta alcanzar su máximo en los años 2005 y 2006, momento en el que se contabilizan más de 159.000 cambios de residencia anuales y se registran tasas superiores al 33\%o (figura 3).

Figura 3

Evolución de la migración intrametropolitana-RMB-1996-2011.

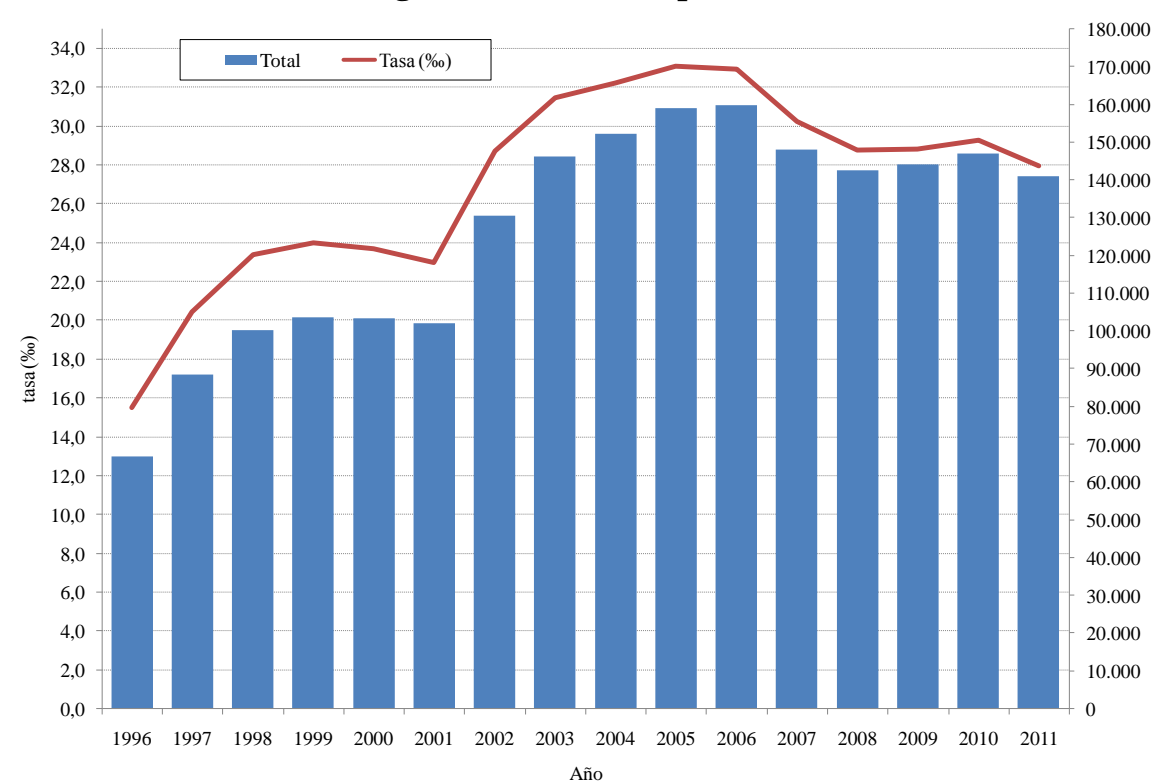

Fuente: Elaboración propia a partir de INE: Estadística de Variaciones Residenciales. Fichero de Microdatos, 1996-2011 y Padrón Municipal de Habitantes, 1996 y Padrón Continuo, 1998-2012.

La coincidencia de estos niveles de máxima movilidad residencial con una coyuntura económica y financiera favorable conducía a pensar que -siguiendo este mismo esquema causal- el impacto de la crisis traería como consecuencia, más o menos inmediata, una acusada reducción de los intercambios migratorios. Esta hipótesis se fundamentaba en el hecho de que una proporción destacada de las migraciones 
intrametropolitanas tenía como principal motivación, hasta el momento, una mejora de la vivienda o del entorno de la misma. Así, según los datos de la Enquesta de condicions de vida $i$ hàbits de la població de Catalunya, un $38 \%$ de los residentes en la RMB que ha cambiado de vivienda lo hace por motivos relacionados con las características de la vivienda o del entorno residencial de la misma, frente a un $34 \%$ que se independiza o sólo un $8,2 \%$ que indica motivos laborales o de formación como su causa. Paralelamente, un 30\% de los entrevistados en un estudio sobre la migración hacia urbanizaciones de la $\mathrm{RMB}^{15}$ demuestra que las características de la vivienda son el principal factor causante de la migración hacia dichos destinos, en especial en lo que se refiere a tamaño, confort y la posibilidad de disponer, por ejemplo, de jardín o piscina. A este porcentaje cabe añadir casi otro $30 \%$, que alude como principal motivo el entorno o el espacio residencial, destacando, concretamente, aspectos como la mejora de la calidad de vida y la tranquilidad. En resumen, se observa que la mayoría de los entrevistados hace más énfasis en los valores de la zona de acogida que en posibles factores de expulsión del lugar que se abandona. Así, la excesiva polución, ruido o problemas de seguridad de las grandes ciudades, que son un motivo de notable peso en la decisión de una migración residencial en otros países ${ }^{16}$, tiene un efecto menor en el contexto español. Nula influencia parecen tener los motivos fiscales, a diferencia de lo que sucede en EE.UU, donde este factor, según señalan Muñiz et al. ${ }^{17}$ , actúa como un claro incentivo en la movilidad residencial. Finalmente, insistir en que las causas laborales son citadas de forma residual como detonante de la migración en la RMB.

En un escenario de crisis como el actual -que afecta directamente a un amplio espectro de las familias de forma más o menos severa-, una hipótesis inicial basada en que las migraciones vinculadas a motivos residenciales disminuirían su presencia gana en solidez. Por un lado, porque la necesidad de los desplazamientos relacionados con la mejora residencial es inferior a la de otras motivaciones, caso de la emancipación o de los motivos laborales, por lo que son movimientos fácilmente aplazables o cancelables sin que afecten de forma decisiva a la vida de las personas. Por otro, por el cambio en el acceso al crédito $^{18}$, puntal básico en el modelo de cambio de residencia habitual en nuestro país, basado en un elevado porcentaje en la compra, frente a alquiler, como indica Leal ${ }^{19}$ a la hora de caracterizar el modelo residencial de los países del sur de Europa. En un plazo muy breve, se pasa de un modelo de fácil acceso a una hipoteca para la compra de una vivienda a un modelo altamente restrictivo, tanto por los criterios para su concesión como por el tipo de interés aplicado. Este aspecto tiene inmediatos efectos en el freno del mercado inmobiliario y se convierte en un factor que acentúa rápidamente el impacto en este sector del cambio de coyuntura ${ }^{20}$. Finalmente, cabe recordar que una de las principales modalidades de migraciones metropolitanas recientes prima la direccionalidad hacia municipios de baja densidad, los cuales se convierten en receptores netos de migración durante etapas anteriores. Como es bien conocido, los costes privados de mantenimiento, es decir, gastos en calefacción, consumo de agua o electricidad e incluso en seguridad y limpieza, o de

\footnotetext{
${ }^{15}$ Pujadas et al., 2009.

${ }^{16}$ Bendixson, 2004

${ }^{17}$ Muñiz et al., 2006.

${ }^{18}$ Brandis, 2010.

${ }^{19}$ Leal, 2009.

${ }^{20}$ Rodríguez-López, 2011.
} 
transporte se incrementan de forma notoria al residir en disperso. En concreto, Henry ${ }^{21}$ estima que vivir en una casa a las afueras de la ciudad cuesta el doble que residir en un piso del centro. Por este motivo, en un momento de restricción generalizada de los ingresos familiares como el actual, las sensibles diferencias en el coste de la vida en función de la elección residencial deberían adquirir mayor protagonismo que las que jugaban en las etapas de crecimiento económico y actuarían como un factor desincentivador de los cambios de residencia hacia los destinos que comportan mayor gasto.

Esta hipótesis inicial, que preveía un efecto directo del cambio en la coyuntura económica en la movilidad residencial, se desvanece o, por lo menos, se cuestiona a la luz de los datos de los últimos años. En un primer momento, la movilidad intrametropolitana parece seguir una evolución paralela a la recesión económica e incluso se adelanta en su manifestación temporal. De este modo, ya en 2007 se aprecia una disminución del tamaño de los flujos que parecía anunciar el final de la etapa de alta movilidad. Sin embargo, los datos de los años siguientes no confirman dicha tendencia inicial: la movilidad entre 2008 y 2011 se estabiliza en valores entre 148.000 y 141.000 migraciones anuales, cifras que significan tasas de un $28-29 \%$ o (ver de nuevo figura 3). La comparación de la movilidad intrametropolitana entre su momento más álgido (año 2006) y el 2011 arroja una diferencia de -18.847 cambios de residencia, es decir, de un $11,8 \%$. Además, la acentuación de la crisis no conlleva una reducción progresiva de los flujos a medida que ésta avanza cronológicamente y los indicadores macroeconómicos básicos empeoran. Por tanto, la vinculación entre crisis y reducción de la migración queda puesta en entredicho, al menos a partir de esta primera visión global. Más allá del efecto en la movilidad general, cabrá buscar, pues, las transformaciones que la crisis conlleva a nivel de composición de los flujos, ya que esta perspectiva arrojará más luz a los verdaderos cambios acaecidos.

\section{¿Somos iguales, somos distintos? Las distintas respuestas de la movilidad frente a la crisis.}

Entre las líneas de investigación que pretende abordar este artículo destaca como tema en el que profundizar el análisis del desigual impacto de la crisis en los distintos colectivos y territorios. Esta línea de trabajo gana en importancia a la luz de los primeros resultados obtenidos, cuando se ha evidenciado que el contexto de crisis tiene un leve efecto en los niveles de movilidad intrametropolitana registrados en la RMB cuando se consideran de forma conjunta y, por tanto, aparece con mayor insistencia la pregunta de si su influencia será desigual en determinados subconjuntos. En este sentido, este epígrafe ahonda en el impacto diferencial del cambio de coyuntura económica según se trate de población de nacionalidad española o extranjera, de acuerdo con el tramo de edad y en los distintos flujos, siempre sin perder de vista las diferencias según grado de compacidad de los municipios que, tal y como se ha demostrado, se revela como una característica determinante en la movilidad metropolitana reciente.

\footnotetext{
${ }^{21}$ Henry, 2007.
} 


\section{Los nuevos actores. La movilidad intrametropolitana de los extranjeros}

Una de las características más destacadas en la evolución de la movilidad residencial en los últimos años en la RMB es la creciente participación de la población de nacionalidad extranjera en este tipo desplazamientos. En términos absolutos, el promedio anual de movimientos intrametropolitanos protagonizados por extranjeros crece de forma notoria, pues pasa de 5.588 durante 1996-2000 a 36.818 en 2001-2007 y alcanza los 53.501 en el período 2008-2011. El resultado de este intenso incremento de los flujos es que los extranjeros pasan de representar el 4,8\% de la migración intrametropolitana durante el período $1996-2001$ al 37,2\% en el período 2008-2011. El valor máximo de participación es el alcanzado en el año 2008, cuando los extranjeros significaron el $41,3 \%$ del flujo migratorio residencial, muy por encima de su peso sobre el total de la población, que en 2012 significaba el 14,5\% de la población metropolitana.

El aumento de la participación de los extranjeros en la movilidad residencial que se produce en general va acompañado de una ampliación de las diferencias entre las distintas categorías municipales según grado de compacidad del municipio. Los municipios clasificados bajo la categoría de compacidad muy alta presentan una participación de extranjeros superior al 50\% entre 2005-2010. En cambio, en los municipios considerados de dispersión máxima son tan sólo el $15 \%$ durante el mismo período (figura 4). Sin embargo, estos son los únicos que mantienen un peso estable de los extranjeros en la inmigración, frente al descenso experimentado en el resto de las categorías a partir de 2008.

Figura 4

Peso proporcional de la población de nacionalidad extranjera sobre la migración intrametropolitana según compacidad/dispersión del municipio. 1996-2011

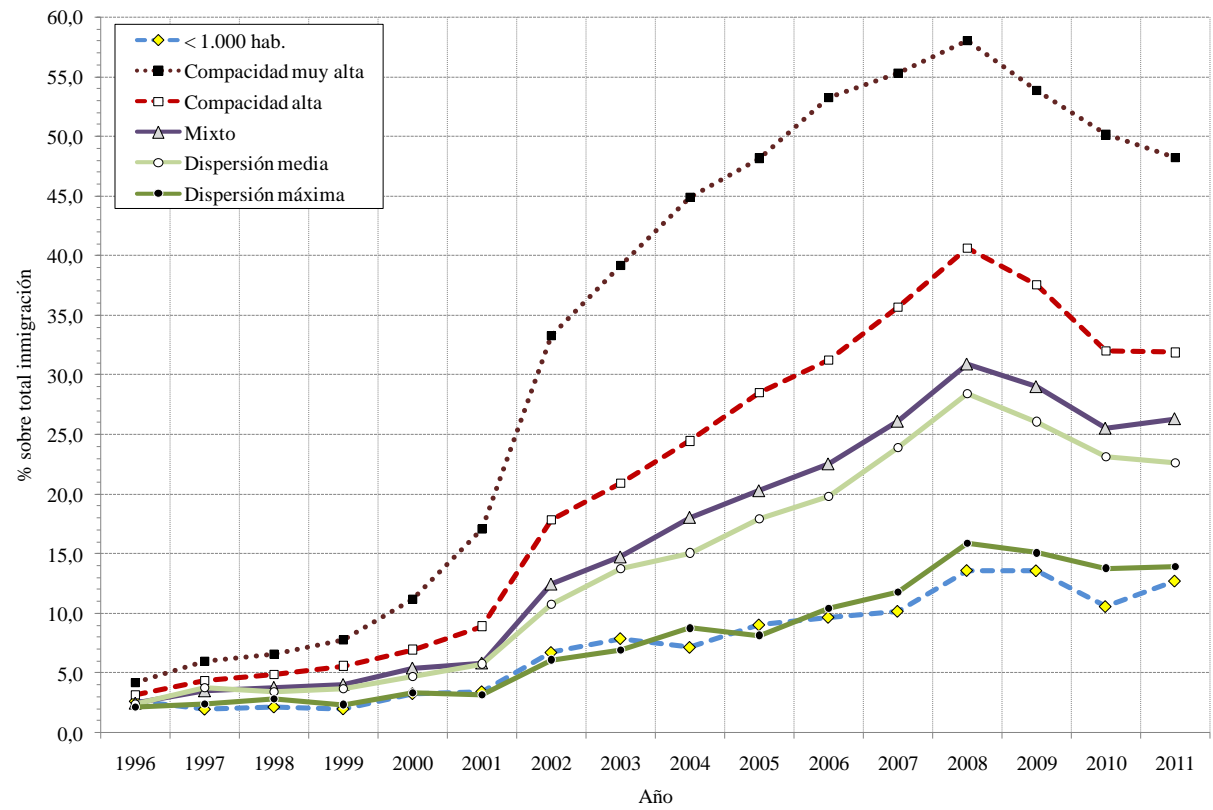

Fuente: Elaboración propia a partir de INE: Estadística de Variaciones Residenciales. Fichero de Microdatos, 1996-2011 y Padrón Municipal de Habitantes, 1996 y Padrón Continuo, 1998-2012.

La movilidad residencial de los extranjeros es extraordinariamente intensa y contrasta en gran medida con la de los españoles (figura 5). Sus tasas de migración 
intrametropolitana aumentan espectacularmente hasta el año 2006 -cuando alcanza su máximo valor $(85,7 \%$ ) - y se sitúa en todo momento en valores muy superiores a los de los españoles. La llegada reciente de la mayoría de población extranjera y la provisionalidad relacionada con las primeras etapas de asentamiento, tanto residencial, laboral o de consolidación familiar explica, en parte, esa mayor movilidad que se extiende a lo largo de todas las edades activas y no sólo en el momento de formación familiar $^{22}$.

Figura 5

Tasas de migración intrametropolitana según nacionalidad y porcentaje de participación de población extranjera sobre el total de la migración intrametropolitana

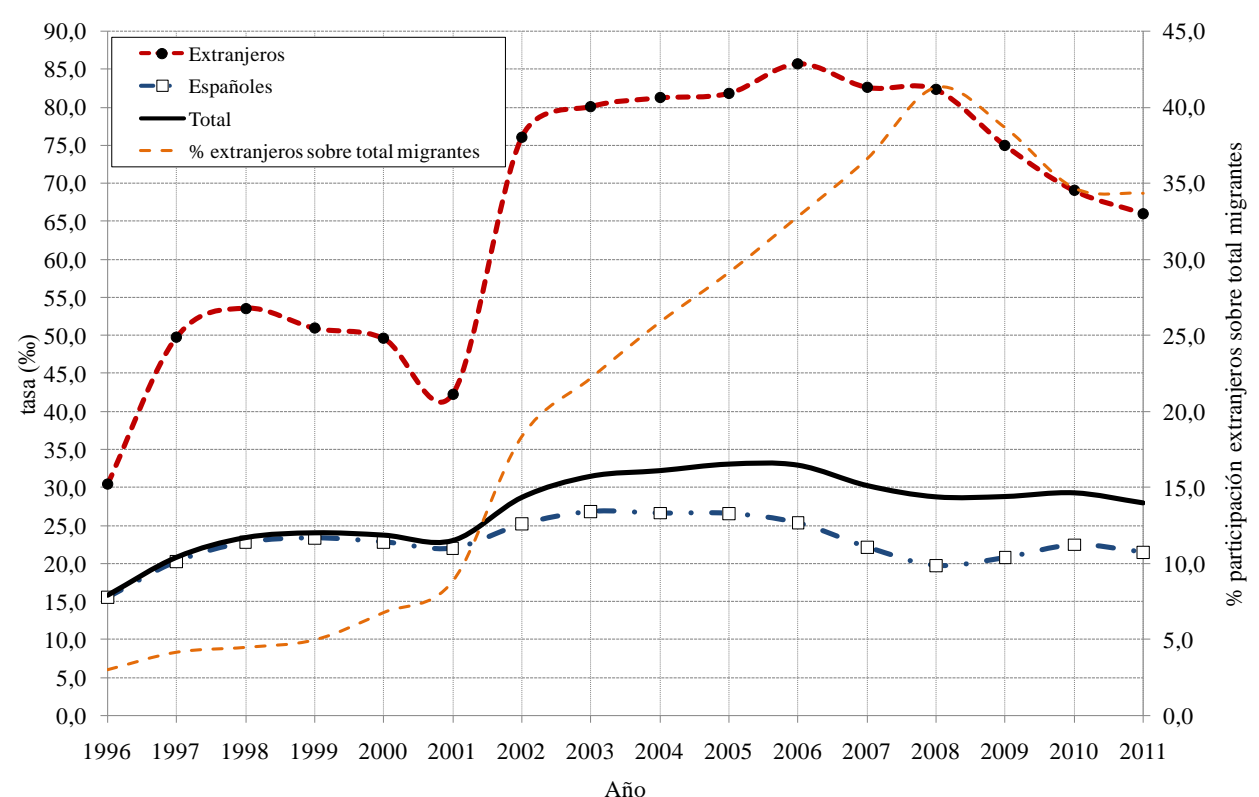

Fuente: Elaboración propia a partir de INE: Estadística de Variaciones Residenciales. Fichero de Microdatos, 1996-2011 y Padrón Municipal de Habitantes, 1996 y Padrón Continuo 1998-2012.

A partir de 2006, la tasa de migración desciende hasta alcanzar el 66\% en 2011, momento en el que la movilidad de los españoles se situaba en un $21 \%$. La pregunta clave en este contexto es incidir si la crisis afecta en mayor medida la movilidad de los españoles o de los extranjeros. De acuerdo con los datos, la merma es mucho más acusada entre los extranjeros, ya que para este colectivo las tasas disminuyen en un $23 \%$ su valor, cuando este mismo cálculo aplicado a la población española arroja como resultado una disminución tan sólo de un 15\%, al pasar de un 25 a un 21\%. En definitiva, la crisis frena en mayor medida la movilidad de los extranjeros que la de los españoles. Un aspecto importante a tener en cuenta en este sentido es el severo empeoramiento de las condiciones laborales de los extranjeros, ya que los indicadores que se refieren a pérdida de empleo, tasa de paro, precarización laboral o grado de acceso a las medidas de protección social frente al deterioro del mercado del trabajo son siempre peores en el caso de esta población. Cabe pensar que precisamente esta desigual situación convierte a los extranjeros en un colectivo más vulnerable en el panorama social actual y, en último término, también tiene su incidencia frenando movilidad residencial. Migraciones vinculadas a una mejora laboral o de la vivienda, siguiendo el proceso de suburbanización iniciado también por los extranjeros en la etapa anterior, se verían así debilitadas en esta etapa de coyuntura económica desfavorable. Finalmente, cabe indicar que la menor llegada de extranjeros a España,

\footnotetext{
${ }^{22}$ Pujadas y Bayona, 2015.
} 
como consecuencia de la crisis, tiene como efecto, a posteriori, una reducción de los cambios de residencia protagonizados por inmigrantes extranjeros, que son especialmente móviles durante los primeros años de asentamiento.

\section{De la movilidad a las movilidades. La importancia de la edad en el comportamiento residencial.}

Los cambios en la movilidad residencial por edades están fuertemente vinculados a los principales eventos del curso de vida: emancipación y formación de un hogar propio, nacimiento de un hijo (primero o sucesivas ampliaciones de la familia), ruptura de la pareja debido a una separación o divorcio, la salida del mercado laboral como consecuencia de la jubilación $\mathrm{y}$, finalmente, la entrada en una situación de dependencia, en mayor o menor grado, causado por el avance en la vejez. Estos eventos, especialmente los primeros y los últimos, tienen un patrón etario muy concreto, de forma que son los que modelan y explican las diferencias de intensidad en la incidencia de la migración, siguiendo los patrones establecidos en los trabajos de Warnes o Rogers ${ }^{23}$.

En el caso de la RMB (figura 6), las pautas de movilidad residencial por edad son muy claras, pues destacan las tasas intensas de migración entre los 25 y 34 años, coincidiendo con el principal momento de formación y ampliación de la familia. En este tramo de edad, las tasas llegan a alcanzar valores superiores al 60\%, concentrando más de un tercio de los cambios residenciales que se registran. Así, en el caso del período de máxima movilidad (2001-2007), éstas llegan a representar un 36,4\% del total de los movimientos. De la misma manera, la intensa movilidad de los adultosgenera una situación de "arrastre" de niños, especialmente acusada en el caso de los menores de 5 años, situación que evidencia la presencia de parejas con hijos pequeños. Tras los máximos registrados entre 25 y 34 años, la movilidad se reduce de forma paulatina, si bien mantiene tasas moderadas, superiores al 30\%o entre los 35 y 44 años. A partir de los 45 años, la movilidad disminuye hasta situarse en tasas muy bajas y en progresivo descenso; únicamente se aprecia un cierto repunte en el tramo de los mayores de 75 años.

El modelo de movilidad de la RMB presenta particularidades de naturaleza diversa, algunas coincidentes con los rasgos propios de la movilidad española, y otras que se explican por su contexto metropolitano específico. En este sentido, cabe apuntar brevemente algunas de estas características. En primer lugar, se aprecia que la movilidad máxima -con tasas superiores al 55\% en los últimos períodos- se localiza en el grupo de edad de 25-29, aunque seguido muy de cerca por el grupo 30-34. En cambio, las pautas de movilidad a edades más jóvenes, caso del grupo 20-24, es bastante inferior, siguiendo el modelo de la movilidad española ${ }^{24}$. A pesar de la contribución de las migraciones de los extranjeros, que se caracterizan por una movilidad de calendario más precoz y mucho más intenso respecto a los autóctonos ${ }^{25}$, las tasas de este colectivo se sitúan veinte puntos por debajo de las registradas en las edades siguientes, es decir, entorno al 40\%o.

\footnotetext{
${ }^{23}$ Warnes, 1992; Rogers, 1990.

${ }^{24}$ Recaño, 2004; García-Coll, 2005.

${ }^{25}$ Pumares et al. 2006 y Recaño, 2002.
} 
Figura 6

Tasas de migración intrametropolitana según edad. RMB-1996-2011

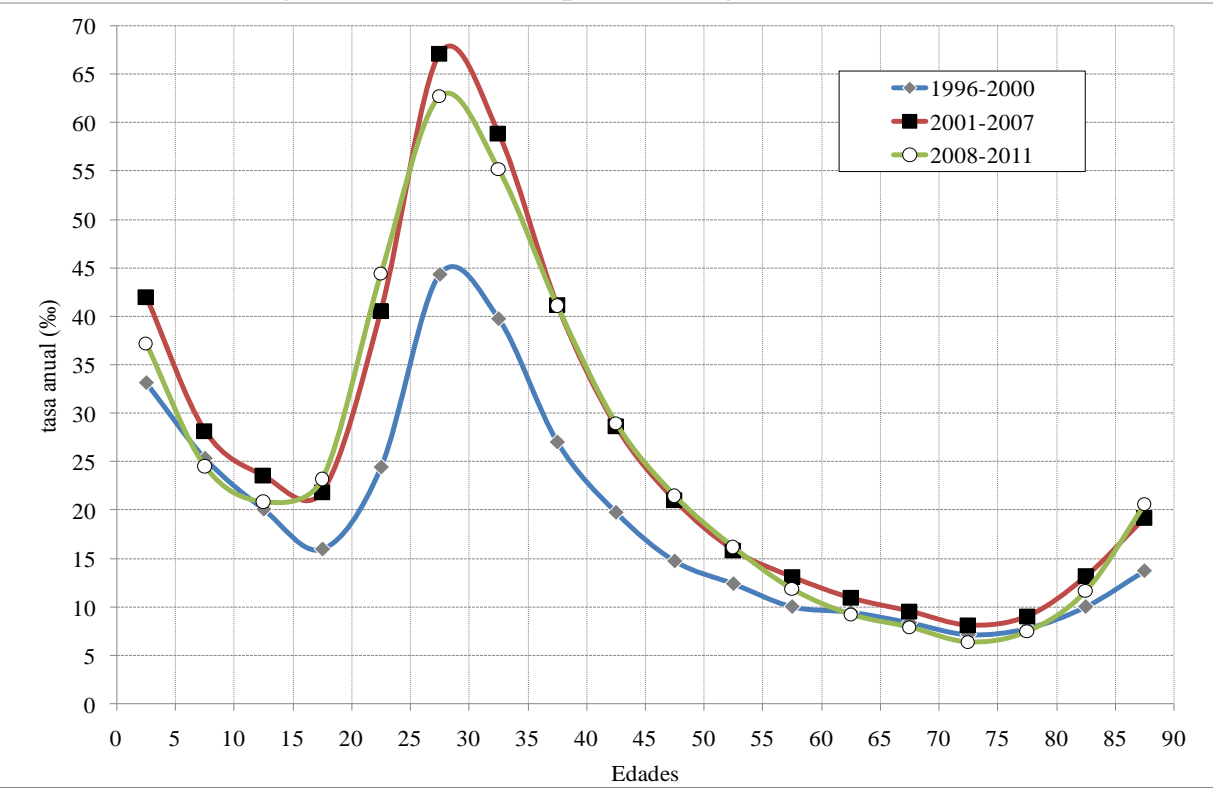

Fuente: Elaboración propia a partir de INE: Estadística de Variaciones Residenciales. Fichero de Microdatos, 1996-2011 y Padrón Municipal de Habitantes, 1996 y Padrón Continuo, 1998-2012.

Esta situación es característica del modelo de movilidad española, consecuencia de un esquema de emancipación mucho más tardío que el presentado por otros países ${ }^{26}$. Otra característica específica es el escaso impacto de la entrada en edad de jubilación en la movilidad: los grupos de edad comprendidos entre los 55 y los 64 años, que recogería la incidencia de las prejubilaciones- o el de 65-69 años -propio de edad legal de jubilación hasta el momento- no lleva asociado un aumento significativo de la movilidad. En cambio, los grupos de edad los 35 y los 44 años presentan tasas que, si bien son sensiblemente menores que las propias de edades de máxima movilidad, pues se sitúan entre un 29 y un 40\%o según sea el período que se observe, muestran un mantenimiento de niveles de movilidad considerables también en los tramos medios de edad. Esta circunstancia refleja el peso de las migraciones causadas por la mejora de la vivienda, que involucra a un segmento más amplio de la población que incluye también etapas más estables del ciclo de vida. En definitiva, la importancia de los movimientos relacionados con la vivienda y el entorno residencial tiene una traducción en la composición por edad. Finalmente, se aprecia un aumento de la movilidad para los mayores de 75 años. En este caso se entremezclan varios determinantes. Por un lado, la pérdida de la independencia residencial de los mayores va, generalmente, acompañada de una movilidad hacia los hogares de los hijos, que, en este caso reproduciría el proceso de suburbanización realizado por los mismos en etapas anteriores. Otro factor que puede incidir es la existencia de una oferta de plazas en residencias en las áreas dispersas de la Región metropolitana, circunstancia que explicaría la movilidad hacia estas zonas de personas de edad avanzada.

La comparación de la movilidad por edad muestra un patrón etario que se repite en los tres períodos analizados. Tras una intensificación de las tasas a todas las edades en 1996-2000, consecuencia del crecimiento general de la movilidad, los dos períodos siguientes se caracterizan por el mantenimiento de un comportamiento muy similar en

\footnotetext{
${ }^{26}$ Miret, 2007.
} 
ambos momentos. De este modo, a pesar de las enormes diferencias en la coyuntura económica, la movilidad no parece resentirse de forma sensible. De hecho, únicamente se aprecia un leve descenso de 6 puntos de las tasas en los grupos de máxima migración, es decir, entre los 25 y los 34 años, y algo más intensa entre 0-14 y los 55 y 84 años. Por su lado, los leves incrementos de las tasas se sitúan entre los 15-24 años. Un factor explicativo de este comportamiento es el aumento de las tasas del grupo 4054 , que aunque porcentualmente es bajo -nunca supera el $2 \%$ - genera un impacto sobre los grupos de jóvenes.

Mayores diferencias en los comportamientos se constatan cuando se comparan las tasas de migración por edad y nacionalidad de las dos grandes etapas de evolución (2001-2007 y 2008-2011). Así, además de comprobar que, como ya se había evidenciado en el epígrafe anterior, la reducción de la movilidad intrametropolitana es mucho más acusada para los adultos extranjeros, se observan algunos contrastes más. En primer lugar, la movilidad intrametropolitana de los españoles se resiente prácticamente en todas las edades; en cambio, en los extranjeros se concentra en el primer tramo de edad, de forma que a partir de los 55 años las variaciones tienen escasa entidad. En segundo lugar, cabe reseñar que la disminución intensa de la movilidad residencial de los extranjeros se mantiene entre los 20 y los 54 años, mientras que en el caso de los españoles la reducción más enérgica se concentra entre $\operatorname{los} 25$ y los 34 años.

Figura 7

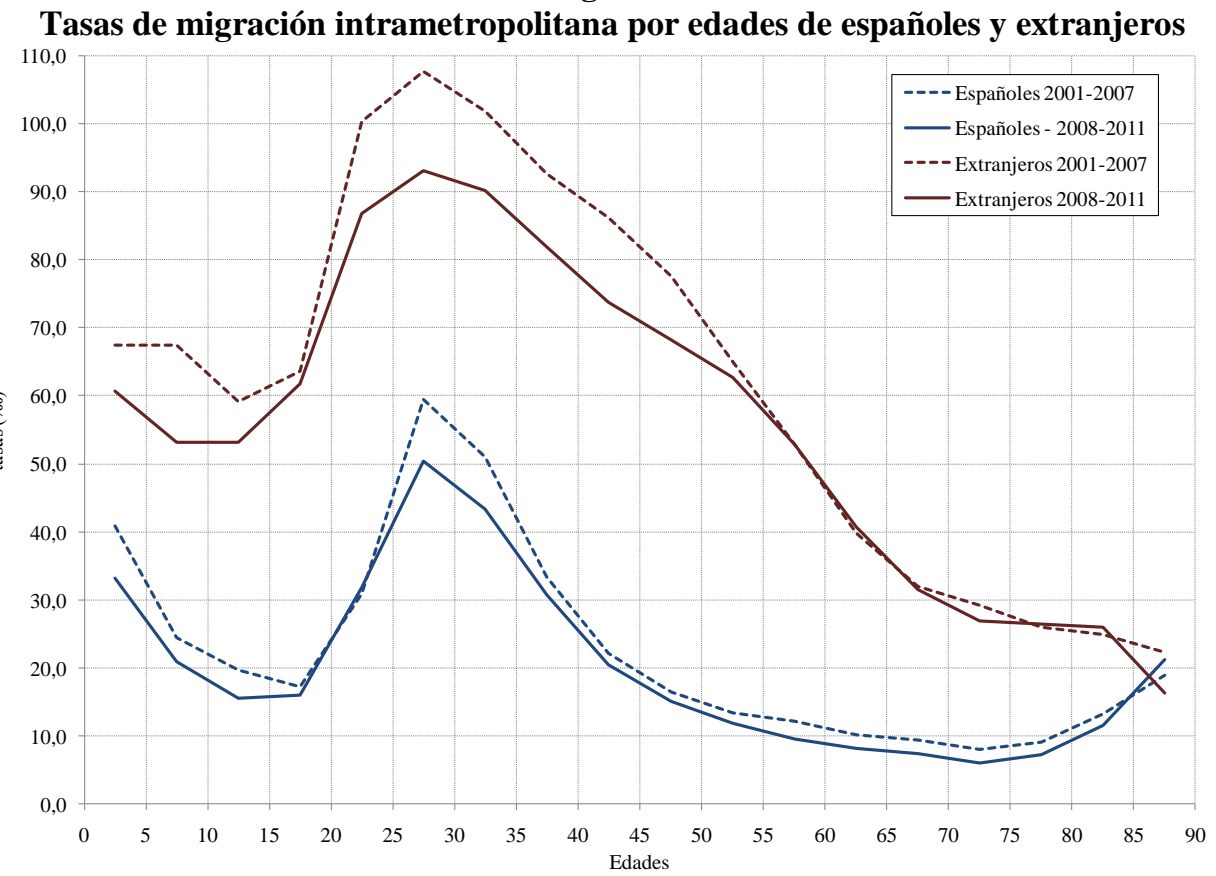

Fuente: Elaboración propia a partir de INE: Estadística de Variaciones Residenciales. Fichero de Microdatos, 2001-2011 y Padrón Continuo, 2001-2012.

Entre los factores que pueden explicar este hecho cabe señalar las características distintivas del mercado laboral de los extranjeros que, a diferencia de lo que sucede con los españoles, ostentan unas elevadas tasas de paro juvenil que no disminuyen con la edad, sino que se mantienen con valores muy acentuadas a lo largo de toda la vida laboral. Esta circunstancia explicaría el sintomático aminoramiento de las tasas de 
movilidad en una franja etaria muy amplia, además de otras estrategias tales como el retorno al país de origen o una nueva migración internacional ${ }^{27}$.

\section{La dimensión territorial del efecto de la crisis, elemento clave para entender los procesos en la RMB.}

Una vez comprobadas las diferencias según nacionalidad y edad cabe adentrarse en la dimensión territorial de las transformaciones, recuperando la perspectiva aportada por la tipología municipal según grado de compacidad. El proceso de redistribución reciente de la población de la RMB confiere a las distintas agrupaciones de municipios un papel muy contrastado y reproduce -al igual que sucedía con el comportamiento del crecimiento de la población en general- la existencia de una relación inversa entre el nivel de compacidad y la tasa de migración neta (figura 8). La única salvedad a esta norma se encuentra en los municipios menores de 1.000 habitantes, ya que estos no siempre ostentan los valores más elevados, sino que hasta 2005 se colocan con índices inferiores a los de máxima dispersión. En definitiva, la disposición general muestra una relación perfecta entre compacidad y migración neta, tal y como los modelos teóricos pronostican ${ }^{28}$.

Figura 8

Tasa de migración neta intrametropolitana según tipología municipal

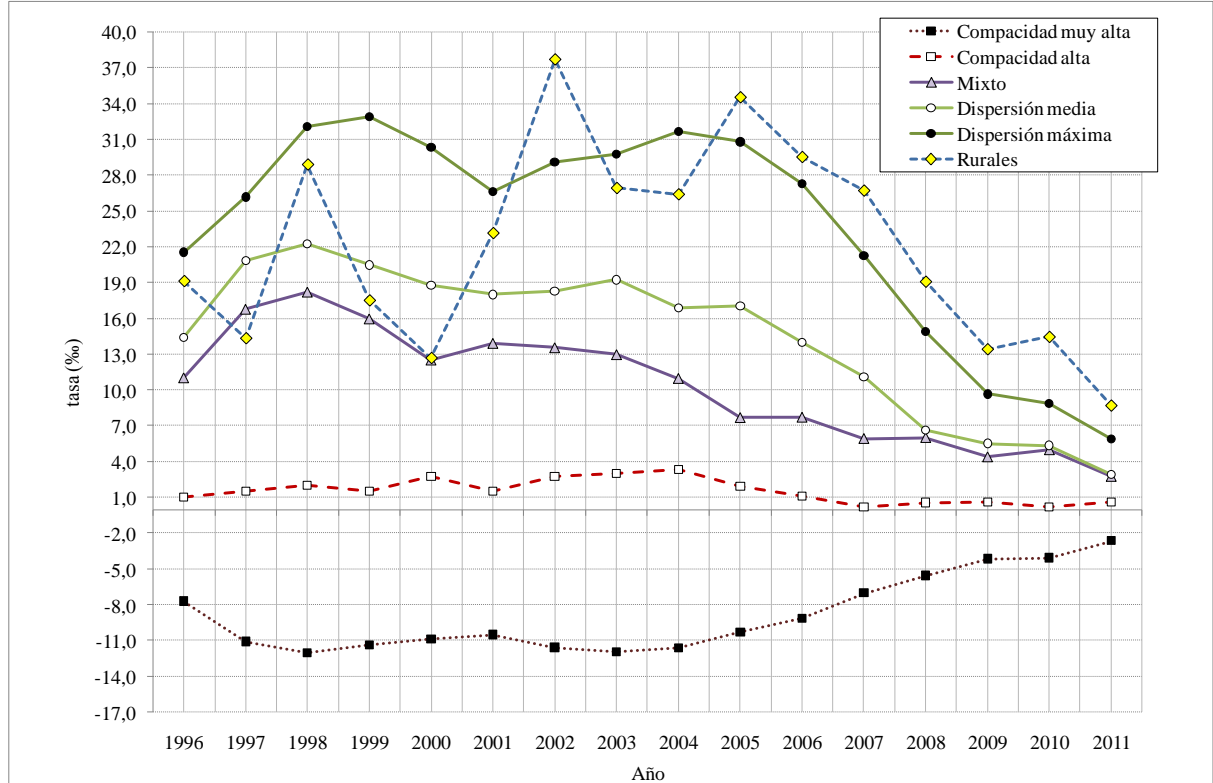

Fuente: Elaboración propia a partir de INE: Estadística de Variaciones Residenciales. Fichero de Microdatos, 1996-2011 y Padrón Municipal de Habitantes, 1996 y Padrón Continuo, 1998-2012.

Un rasgo común de la movilidad residencial de la RMB durante el período estudiado es que los municipios de muy alta compacidad se mantienen como los principales alimentadores de las corrientes que se dirigen al resto de la Región ${ }^{29}$, pues son los únicos con una tasa de migración neta de signo negativo durante todo el período. El comportamiento opuesto es el desempeñado por los municipios dispersos, los cuales presentan unas intensas tasas de migración neta durante la práctica totalidad del

\footnotetext{
${ }^{27}$ Reher et al, 2011, Domínguez Mujica et al, 2010.

${ }^{28}$ Fielding, 1982.

${ }^{29}$ Bayona y López-Gay, 2011.
} 
período analizado, muy elevadas en el momento de máxima expresión de la dispersión y, sobre todo, en el caso de los municipios de dispersión máxima. Mucho más modesto es el papel jugado por las categorías de alta compacidad y los mixtos, conjuntos que registran tasas de migración neta de signo positivo pero de valores moderados, cercanos a cero en el caso de los de alta compacidad y ligeramente más elevados en el caso de los segundos. Caso aparte es el de los municipios menores de 1.000 habitantes, que se caracterizan por su papel cambiante -inicialmente, tasas próximas a la media pero que se incrementan sensiblemente con el paso del tiempo-, evidencia del nuevo papel desempeñado por estos al incorporarse a la dinámica de dispersión como áreas alternativas de crecimiento.

A pesar del papel más o menos estable desempeñado por cada una de las categorías territoriales señaladas, la observación de la evolución temporal permite constatar las modificaciones experimentadas a partir de 2007, las cuales están estrechamente relacionadas con la incidencia de la crisis. En primer lugar, porque se aprecia una tendencia compartida a la disminución de las tasas para todas las categorías. En segundo lugar, porque se produce una reducción mayor en los municipios que tenían unas ganancias migratorias de mayor entidad frente a una menor incidencia en los que tienen valores menos pronunciados. El resultado final es una aproximación de las tasas de migración neta de las distintas categorías que rompe con la dispersión de valores propia del período precedente. Con el fin de comprender mejor las razones de estos nuevos comportamientos es necesario un análisis más afinado de lo sucedido, abandonando la perspectiva de la migración neta y recabando información de los flujos de inmigración y emigración por separado (figura 9).

Esta nueva perspectiva ilustra cómo los municipios de muy alta compacidad, a pesar de seguir registrando una migración neta de signo negativo, tienen en esta etapa más reciente una migración neta que se aleja de valores extremos (próximos a los $-11 \%$ alcanzados entre 2002 y 2004), para situarse en niveles más discretos $(-2,6 \%$ en 2011). Esta situación es consecuencia de una ligera disminución de la emigración -que pasa de un máximo del 32\%o en 2005 y 2006 a un mínimo de un 26\%o en 2011- y, sobre todo, de un incremento de la inmigración, que abandona los mínimos inferiores al $11 \%$ alcanzados antes de 2001 para estabilizarse en tasas de un $23 \%$ a partir de 2006 hasta la actualidad. Se trata, por tanto, de una situación novedosa, fundamentada en un aumento de la inmigración intrametropolitana y un freno de la emigración. Por su lado, los municipios de alta compacidad se estabilizan alrededor de 2008 en tasas en torno al 25\%o, mostrando un ligero descenso respecto $30 \%$ ontre 2003 y 2006, tanto en su inmigración como emigración. Cabe insistir que los municipios compactos son los únicos que no registran una caída acusada de la inmigración en el tramo más reciente, sino que logran recuperar ligeramente sus índices desde principios de este siglo hasta $2006 \mathrm{y}$, a partir de ese momento, estabilizan sus ratios de entrada. Este incremento de la inmigración reforzaría las hipótesis de reurbanización que lanzan algunos autores como López-Gay, Champion o Bourne ${ }^{30}$, o de la convivencia de reurbanización y dispersión, en el sentido apuntado por trabajos como los de Nel·lo o Kabisch y Haase ${ }^{31}$ y daría pie a las teorías que resaltan los valores de la centralidad en etapas de recesión económica.

\footnotetext{
${ }^{30}$ López-Gay, 2011; Champion, 2001; o Bourne, 1996.

${ }^{31} \mathrm{Nel} \cdot$ lo, 2007; o Kabisch y Haase, 2011.
} 
Figura 9

Componentes del crecimiento migratorio según tipologia residencial
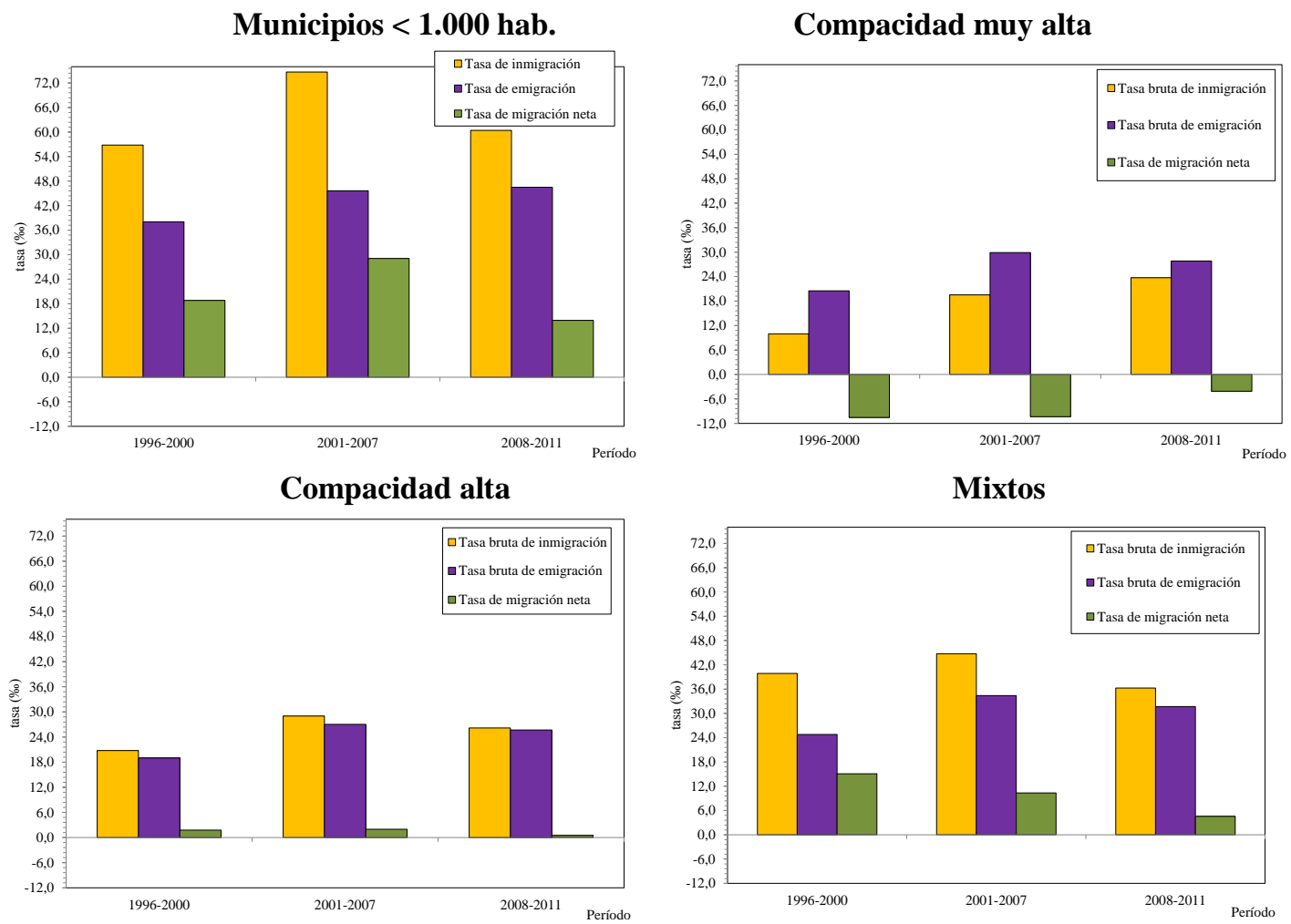

Dispersión media
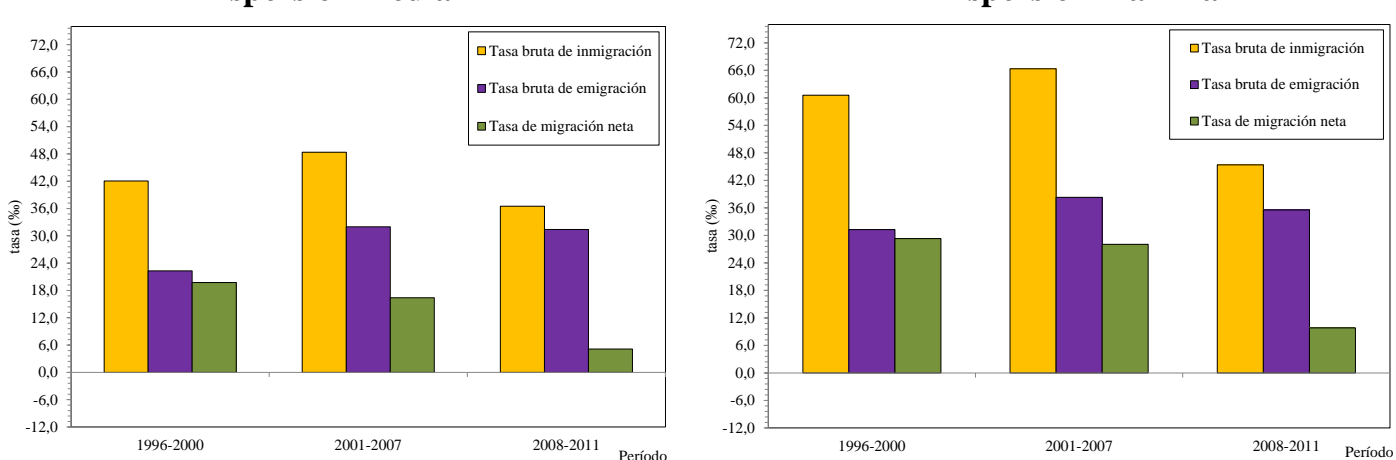

Fuente: Elaboración propia a partir de INE: Estadística de Variaciones Residenciales. Fichero de Microdatos, 1996-2011 y Padrón Municipal de Habitantes, 1996 y Padrón Continuo 1998-2012.

El efecto más claro de la crisis se produce en los municipios dispersos, en concreto en su inmigración, que experimenta ahora un acentuado descenso. El factor clave de su transformación es un contundente aminoramiento de la inmigración, frente a tasas muy estables de emigración. Así, los municipios de dispersión media inician un proceso de descenso progresivo de su inmigración que se anticipa a los de dispersión máxima. Este fenómeno refleja el proceso de saturación de los municipios de dispersión media, que tienden a colmatarse y encarecerse, siendo desplazados en el mercado por los municipios de dispersión máxima. Cabe recordar que estos municipios registraron tasas de inmigración cercanas al 30\%o entre 1998 y 2005, muestra evidente de la intensidad del proceso y de la duración del mismo. 
Las tasas de migración neta intrametropolitana por edad según tipología diseñada en función de su nivel de compacidad actúan como informadoras de la especialización migratoria de cada uno de los grupos de municipios (figura 10) y de las transformaciones experimentadas de forma reciente (figura 11), de manera que completan el análisis llevado a cabo hasta el momento.

\section{Figura 10}

Tasas de migración neta por edades según tipología de municipios (1996-2011)
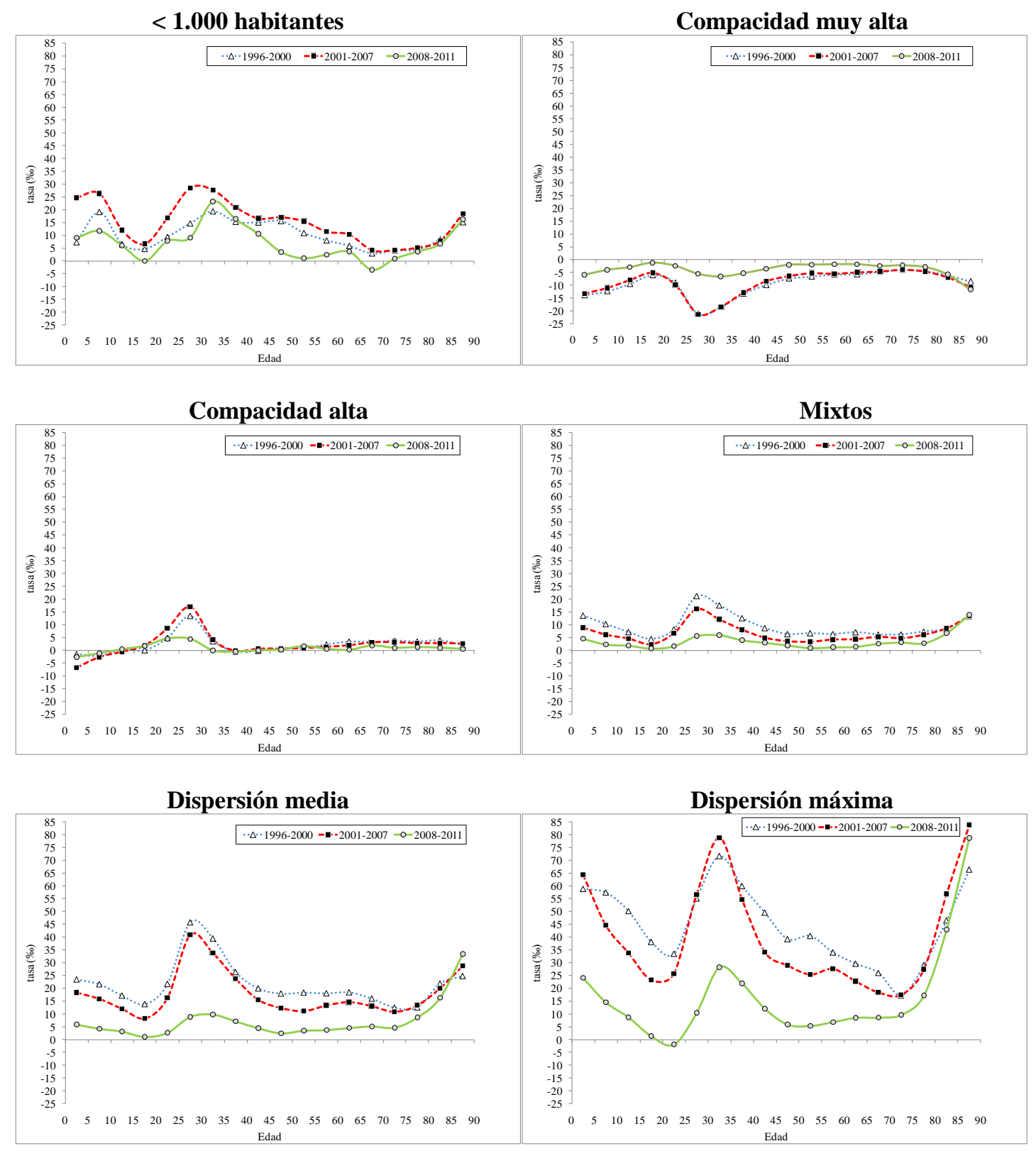

Fuente: Elaboración propia a partir de INE: Estadística de Variaciones Residenciales. Fichero de Microdatos, 1996-2011 y Padrón Municipal de Habitantes, 1996 y Padrón Continuo, 1998-2012

A pesar de las modificaciones comentadas anteriormente, los municipios de muy alta compacidad mantienen su condición de emisores netos de población en relación con el resto del territorio metropolitano. Sus tasas son negativas para todas las edades y para los tres períodos, por lo que su talante todavía emigratorio no puede ser cuestionado. No obstante, es cierto que en la etapa más reciente, la negatividad de sus tasas se 
reduce, registrando una sensible merma en los adultos-jóvenes y en las edades centrales. Estos municipios presentan una trayectoria contrastada en su comportamiento por edad, con un claro aumento de la inmigración antes de los 69 años que cambia de signo a partir de dicho momento. Paralelamente, sus tasas de emigración apenas varían en las edades centrales, con un ligero aumento en la franja 15-24 y disminución para el de 25-34. Parece, por tanto, que la crisis está reajustando la emigración de estas áreas en el caso de los adultos-jóvenes pues, de hecho, migran de forma conjunta como una única unidad familiar.

Figura 11

Variación en las tasas de inmigración y emigración entre 2001-2007 y 2008-2011 (\%)
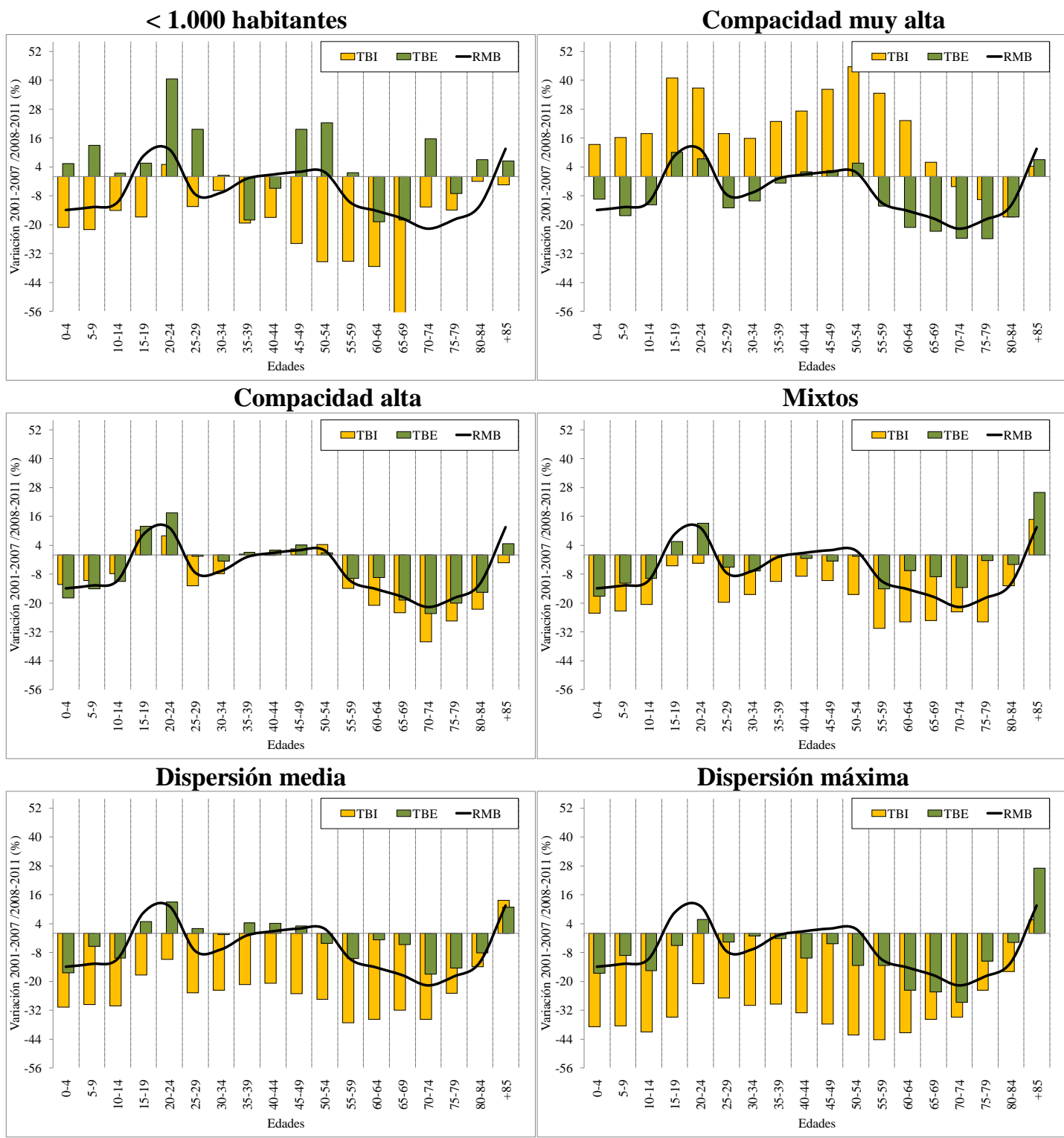

Fuente: Elaboración propia a partir de INE: Estadística de Variaciones Residenciales. Fichero de Microdatos, 1996-2011 y Padrón Municipal de Habitantes, 1996 y Padrón Continuo 1998-2012.

Lo que no puede comprobarse a partir de los datos disponibles es si el descenso de la tasa es consecuencia del retraso en la edad de emancipación o bien sugiere que, gracias a las nuevas circunstancias como, por ejemplo, el reajuste del precio de los alquileres, 
una parte de los jóvenes que en otros momentos hubiese emigrado puede ahora permanecer en ella. En cambio, el descenso más severo de las salidas desde los muy compactos cabe buscarla en el grupo de 55 y más años. En este sentido, todo apunta a que la ciudad más compacta se convierte en un valor en alza en momentos de crisis. Los valores de su centralidad, en aspectos como la reducción del coste de la vida o en el transporte (por su mejor conectividad y oferta de transporte público) y las dificultades para poder trasladarse, por ejemplo, a áreas dispersas -en especial para aquellos que no cuentan con una vivienda ya adquirida- frenan la emigración en estas edades.

Por su lado, los municipios dispersos presentan ganancias netas a todas las edades, en las que sobresalen las tres etapas claves. En primer lugar, destacan por la intensidad de sus tasas las edades propias de la emancipación y formación de la familia (25-39 años) y el arrastre en la población infantil propio de la migración familiar de parejas con hijos. Cabe señalar que en estos municipios sí es evidente el incremento de las tasas asociadas al momento de jubilación en el período 2001-2007, diferencia que no se apreciaba en el análisis agregado de la movilidad intrametropolitana y que desaparece en la etapa más reciente. Finalmente, estos se configuran como espacios receptores también de personas mayores, con tasas que se incrementan de forma ostensible a partir de los 75 años. Este patrón se repite tanto en los municipios de dispersión media como de dispersión máxima, si bien en los segundos se manifiestan con mayor intensidad. La huella de la crisis en estos municipios se aprecia de forma diáfana, con una drástica reducción de las tasas para todas las edades, excepto para el grupo de $85 \mathrm{y}$ más. El aumento de la emigración para los mayores de 85 años podría relacionarse con un proceso de reurbanización de personas de edad y estaría estrechamente relacionado con la acentuación de la inmigración para ese mismo tramo de edad en el grupo de compacidad muy alta, una de las hipótesis avaladas por los análisis empíricos en otros países $^{32}$. Según estos preceptos, se produce un proceso de dispersión residencial en las edades próximas a la jubilación y protagonizadas por los llamados viejos-jóvenes. Pero, en cambio, también se detecta un flujo contrario de vuelta a la ciudad para los de mayor edad, coincidiendo con el aumento de la dependencia y una nueva valoración de la centralidad y de la proximidad a los servicios. Por tanto, dada su complejidad, este caso necesita ser objeto de un estudio específico que se aleja del tema central de este trabajo.

A la luz de los datos es necesario repensar el papel de los municipios dispersos en un momento de crisis económica como el actual. A pesar de la rotunda merma de las tasas registradas, la inmigración de los municipios de dispersión máxima y media son, con diferencia, todavía las más elevadas y sigue albergando saldos positivos en los intercambios migratorios para todas las edades. ¿Cómo explicar esta pervivencia de las corrientes que se dirigen hacia municipios dispersos?. En primer lugar, es innegable la continuidad de una modalidad de migración residencial que sigue el estándar clásico, valorando las ventajas residenciales de la oferta en municipios dispersos, en especial entre las parejas jóvenes con hijos pequeños o para aquellos que se jubilan. En este caso, la disponibilidad de una vivienda, bien adquirida con anterioridad o bien de compra reciente, aprovechando una importante rebaja de los precios debido a un exceso de oferta de viviendas en relación con la demanda, sería un factor que contribuiría a la toma de decisión. Este colectivo asume el coste diferencial de la vida

\footnotetext{
${ }^{32}$ Champion, 2001.
} 
en disperso y puede hacerle frente, dada su situación particular. Sin embargo, también puede responder a un cambio en las opciones residenciales de una parte de la población de la $\mathrm{RMB}$, en este caso muy vinculada a la acción de la crisis y las estrategias familiares para afrontarla. Una primera estrategia se basaría en la transformación de segundas residencias en viviendas principales para los propios propietarios o para los hijos de los mismos que, a la hora de enfrentarse a la emancipación, verían con buenos ojos el acceso a una vivienda en un municipio de estas características si ésta es cedida de forma gratuita o con un coste menor al del mercado por sus progenitores. La transformación de la segunda en primera residencia es una práctica identificada y analizada por López Colás et al. y por Pozo y Rodríguez $^{33}$. Una segunda estrategia se basa en transformar una segunda residencia, situada en municipios dispersos, en primera, con el fin de vender o alquilar su hasta el momento vivienda principal ubicada en un municipio de alta o muy alta compacidad. Las características recientes del mercado inmobiliario hacen más fácil la venta o alquiler de un inmueble en un área compacta que en una dispersa, por lo que las familias con problemas económicos que cuentan con dos viviendas de índole distinta pueden optar por cambiar su lugar de residencia habitual hacia la menos atractiva para el mercado.

Finalmente, los municipios dispersos presentan tasas de migración neta destacadas para el segmento de población de más edad que, además, es uno de los que presenta un menor aminoramiento de su inmigración. Llama la atención el proceso de dispersión urbana de las personas mayores, el cual necesitaría de un estudio más detallado, con el fin de profundizar si, tal y como se comentado anteriormente, se trata de personas que se dirigen hacia viviendas colectivas (centros residenciales ubicados en estos municipios) o bien inician un proceso de reagrupación familiar hacia las residencias de los hijos.

\section{Un mismo escenario, unas pautas distintas. El papel de la crisis en la direccionalidad de los flujos.}

Un último elemento de reflexión sobre el efecto de la crisis en los intercambios migratorios intrametropolitanos viene de la mano del estudio de la direccionalidad de los flujos. En primer lugar, si se toma como referencia la tasa de migración neta anual intrametropolitana a escala municipal, es evidente la ruptura que este nuevo período infringe respecto a la situación de las dos etapas anteriores (figura 12).

De este modo, queda claro que durante los dos primeros períodos, existe una clara jerarquía en la movilidad residencial. Esta jerarquía no viene determinada tanto por el signo positivo o negativo de los saldos migratorios de los mismos, como por la intensidad de las ganancias netas, que diferencia municipios con tasas de migración neta muy elevadas, intermedias o bajas. Esta jerarquía se mantiene bastante estable durante 1996-2000 y 2001-2007. En cambio, la diferenciación territorial se diluye en el período más reciente, caracterizado por la desaparición de los focos con tasas de mayor intensidad (que se ve reducido de 63 a 5 casos) y la instalación de un $67 \%$ de los municipios en tasas positivas inferiores a un $15 \%$. La mayor estabilidad proviene, en este caso, de los municipios con balance migratorio negativo, que si bien se

\footnotetext{
${ }^{33}$ López Colás et al., 2007; y por Pozo y Rodríguez, 2006.
} 
incrementan de 18 en 2001-2007 a 27, en 2008-2011, mantienen una disposición territorial bastante similar. Barcelona ciudad y los municipios de la primera corona metropolitana son los que configuran el eje central de este decrecimiento, a los que se suman ya en el último período los municipios más periféricos de la RMB. Cabe recordar que, a pesar de las rotundas diferencias en los resultados, el número de movimientos intrametropolitanos registrados en los dos últimos períodos es muy similar en términos de movimientos, ya que en ambos casos es cercano a los 143.000 migraciones anuales. En cambio, a pesar de las afinidades en la cartografía resultante, el período 1996-2000 moviliza un promedio de 92.000 migrantes intrametropolitanos anuales frente a los casi 143.000 que se desplazan anualmente entre 2001-2007. De nuevo el cambio de escala y el tratamiento agregado de los municipios según su grado de compacidad ayudará a esclarecer las alteraciones del modelo previo estrechamente relacionadas con el efecto de la crisis.

Figura 12

Tasas de migración neta. Municipios RMB. 1996-2011

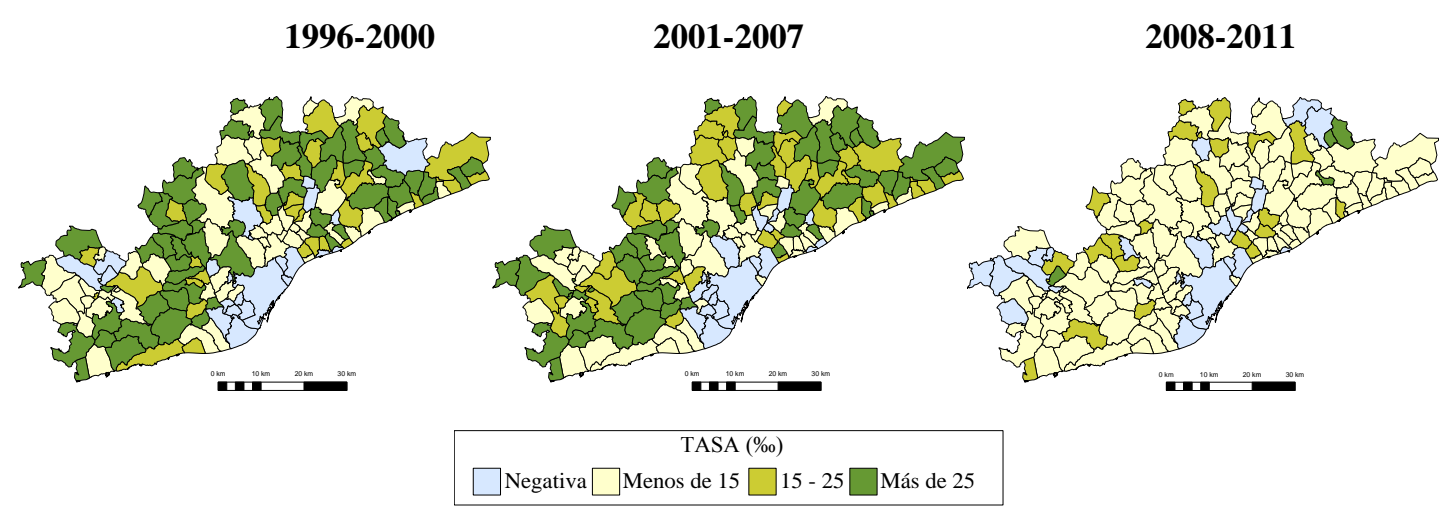

Fuente: Elaboración propia a partir de INE: Estadística de Variaciones Residenciales. Fichero de Microdatos, 1996-2011 y Padrón Municipal de Habitantes, 1996 y Padrón Continuo, 1998-2012.

Cuadro 4

Evolución porcentual de los flujos migratorios según el lugar de destino.

\begin{tabular}{|c|c|c|c|c|c|c|c|}
\hline & $\begin{array}{c}\text { Dispersión } \\
\text { máxima }\end{array}$ & $\begin{array}{c}\text { Dispersión } \\
\text { media }\end{array}$ & Mixto & $\begin{array}{c}\text { Compacidad } \\
\text { alta } \\
\end{array}$ & $\begin{array}{c}\text { Compacidad } \\
\text { muy alta }\end{array}$ & < 1.000 hab. & Total \\
\hline \multicolumn{8}{|l|}{ Españoles } \\
\hline $1996-2000$ & 14,29 & 15,46 & 24,70 & 21,63 & 23,24 & 0,68 & 100,00 \\
\hline 2001-2007 & 15,50 & 15,72 & 23,12 & 21,52 & 23,28 & 0,87 & 100,00 \\
\hline 2008-2011 & 13,49 & 14,05 & 21,55 & 20,74 & 29,25 & 0,93 & 100,00 \\
\hline \multicolumn{8}{|l|}{ Extranjeros } \\
\hline $2001-2007$ & 3,93 & 8,37 & 14,02 & 20,17 & 53,29 & 0,22 & 100,00 \\
\hline $2008-2011$ & 3,92 & 7,93 & 14,08 & 19,22 & 54,62 & 0,23 & 100,00 \\
\hline \multicolumn{8}{|l|}{ Total } \\
\hline $1996-2000$ & 13,97 & 15,28 & 24,48 & 21,72 & 23,88 & 0,67 & 100,00 \\
\hline 2001-2007 & 12,51 & 13,82 & 20,77 & 21,17 & 31,03 & 0,70 & 100,00 \\
\hline $2008-2011$ & 9,93 & 11,77 & 18,77 & 20,17 & 38,69 & 0,67 & 100,00 \\
\hline
\end{tabular}

Fuente: Elaboración propia a partir de INE: Estadística de Variaciones Residenciales. Fichero de Microdatos, 1996-2011

El análisis de las relaciones migratorias entre municipios compactos, mixtos o dispersos se realizará a partir de las matrices según lugar de origen y de destino para los tres períodos analizados (cuadro 4). 
La evolución desde período 1996-2000 hasta el último 2008-2011 está marcada por el aumento del destino migratorio hacia los municipios más compactos y la reducción de las corrientes migratorias hacia los municipios de dispersión media y máxima. Los de compacidad alta han pasado de captar el 23,9\% de los cambios residenciales intermunicipales en el quinquenio 1996-2000 al 38,7\% en el período más reciente. Por su parte, los municipios de dispersión media han reducido su atracción desde un $15,3 \%$ al $11,7 \%$ y los de dispersión máxima del 14\% al 10\%. De modo que, a pesar de que las tasas de crecimiento de la población y las tasas de inmigración siguen siendo más elevadas para los dos grupos de municipios dispersos, su capacidad de atracción está retrocediendo continuamente.

Los resultados muestran la clara preferencia de la población extranjera por los destinos compactos que ya se aprecia de forma rotunda desde 2001. El poder de atracción de los municipios compactos para los extranjeros se puede relacionar con unas mayores oportunidades para la búsqueda de empleo y con un parque de viviendas que permite asumir esta población recién llegada con un stock de alquiler, que es el régimen mayoritario de tenencia entre los no nacionales ${ }^{34}$. No obstante, la novedad en la etapa más reciente se encuentra en el incremento del atractivo de estas áreas también para los españoles. Sin ninguna duda, el principal elemento explicativo de esta tendencia es el impacto de la grave crisis financiera-económica especialmente desfavorable para las opciones residenciales de baja densidad.

Cuadro 5

Evolución porcentual de los flujos migratorios según el lugar de origen

\begin{tabular}{|c|c|c|c|c|c|c|c|}
\hline & $\begin{array}{l}\text { Dispersión } \\
\text { máxima }\end{array}$ & $\begin{array}{l}\text { Dispersión } \\
\text { media }\end{array}$ & Mixtos & $\begin{array}{c}\text { Compacidad } \\
\text { alta }\end{array}$ & $\begin{array}{l}\text { Compacidad } \\
\text { muy alta }\end{array}$ & $<1.000$ hab. & Total \\
\hline \multicolumn{8}{|l|}{ ESPAÑOLES } \\
\hline $1996-2000$ & 7,22 & 8,03 & 15,02 & 19,95 & 49,35 & 0,43 & 100,00 \\
\hline $2001-2007$ & 8,44 & 9,89 & 17,19 & 21,01 & 42,96 & 0,49 & 100,00 \\
\hline 2008-2011 & 10,17 & 11,76 & 18,40 & 20,93 & 38,06 & 0,68 & 100,00 \\
\hline \multicolumn{8}{|c|}{ EXTRANJEROS } \\
\hline $1996-2000$ & 7,05 & 9,63 & 19,06 & 17,99 & 45,46 & 0,81 & 100,00 \\
\hline $2001-2007$ & 3,70 & 6,96 & 12,42 & 16,03 & 60,64 & 0,25 & 100,00 \\
\hline 2008-2011 & 3,74 & 7,35 & 13,04 & 17,86 & 57,77 & 0,23 & 100,00 \\
\hline \multicolumn{8}{|l|}{ TOTAL } \\
\hline $1996-2000$ & 7,21 & 8,11 & 15,22 & 19,85 & 49,16 & 0,45 & 100,00 \\
\hline $2001-2007$ & 7,22 & 9,14 & 15,96 & 19,73 & 47,53 & 0,43 & 100,00 \\
\hline 2008-2011 & 7,77 & 10,12 & 16,40 & 19,79 & 45,40 & 0,51 & 100,00 \\
\hline
\end{tabular}

Fuente: Elaboración propia a partir de INE: Estadística de Variaciones Residenciales. Fichero de Microdatos, 1996-2011.

Por otro lado, se puede observar cuál ha sido la migración según el lugar de origen, a partir de los datos del cuadro 5. La emigración desde los municipios de mayor compacidad se ha reducido desde un $49,1 \%$ en el primer período al $45,4 \%$ en el último. Este es el cambio más representativo en comparación con los restantes clúster de municipios. Así pues, en los otros grupos, las proporciones de salidas aumentan ligeramente (en los de dispersión media y en los mixtos) o bien se mantienen con pocas variaciones (en los de compacidad alta y en los de dispersión máxima).

\footnotetext{
${ }^{34}$ Reher, 2008.
} 
Cuadro 6

Comparación de los flujos migratorios según el lugar de origen y destino. 2001-2007 y 2008-2011

\begin{tabular}{|c|c|c|c|c|c|}
\hline \multirow[b]{2}{*}{ Origen/destino } & \multicolumn{2}{|c|}{$\begin{array}{c}\text { Número de migrantes } \\
\text { (promedio anual) }\end{array}$} & \multirow{2}{*}{$\begin{array}{c}\text { Variación 2001-07 } \\
/ 2008-11 \\
(\%) \\
\end{array}$} & \multicolumn{2}{|c|}{$\%$ sobre total } \\
\hline & 2001-2007 & 2008-2011 & & 2001-2007 & 2008-2011 \\
\hline $\mathrm{De}<1.000$ hab . a $<1.000$ hab. & 37 & 34 & $-8,1$ & 0,03 & 0,02 \\
\hline De compacto a $<1.000$ hab. & 419 & 354 & $-15,5$ & 0,29 & 0,25 \\
\hline De mixto a $<1.000$ hab. & 295 & 299 & 1,3 & 0,21 & 0,21 \\
\hline De disperso a $<1.000$ hab. & 249 & 274 & 9,8 & 0,17 & 0,19 \\
\hline De $<1.000$ hab. a compacto & 186 & 231 & 23,8 & 0,13 & 0,16 \\
\hline De compacto a compacto & 52.032 & 58.876 & 13,2 & 36,49 & 40,96 \\
\hline De mixto a compacto & 11.564 & 13.220 & 14,3 & 8,11 & 9,20 \\
\hline De disperso a compacto & 10.648 & 12.278 & 15,3 & 7,47 & 8,54 \\
\hline De $<1.000$ hab. a mixto & 186 & 225 & 20,7 & 0,13 & 0,16 \\
\hline De compacto a mixto & 20.570 & 17.275 & $-16,0$ & 14,43 & 12,02 \\
\hline De mixto a mixto & 3.679 & 3.717 & 1,0 & 2,58 & 2,59 \\
\hline De disperso a mixto & 5.177 & 5.756 & 11,2 & 3,63 & 4,00 \\
\hline De $<1.000$ hab. a disperso & 202 & 250 & 23,8 & 0,14 & 0,17 \\
\hline De compacto a disperso & 22.874 & 17.185 & $-24,9$ & 16,04 & 11,96 \\
\hline De mixto a dispersos & 7.219 & 6.341 & $-12,2$ & 5,06 & 4,41 \\
\hline De disperso a disperso & 7.244 & 7.414 & 2,3 & 5,08 & 5,16 \\
\hline Total & 142.583 & 143.727 & 0,8 & 100,00 & 100,00 \\
\hline
\end{tabular}

Fuente: Elaboración propia a partir de INE: Estadística de Variaciones Residenciales. Fichero de Microdatos, 1996-2011.

Figura 13

Variación de los flujos según origen y destino en cifras relativas: periodos 2001-2007 y 2008-2011

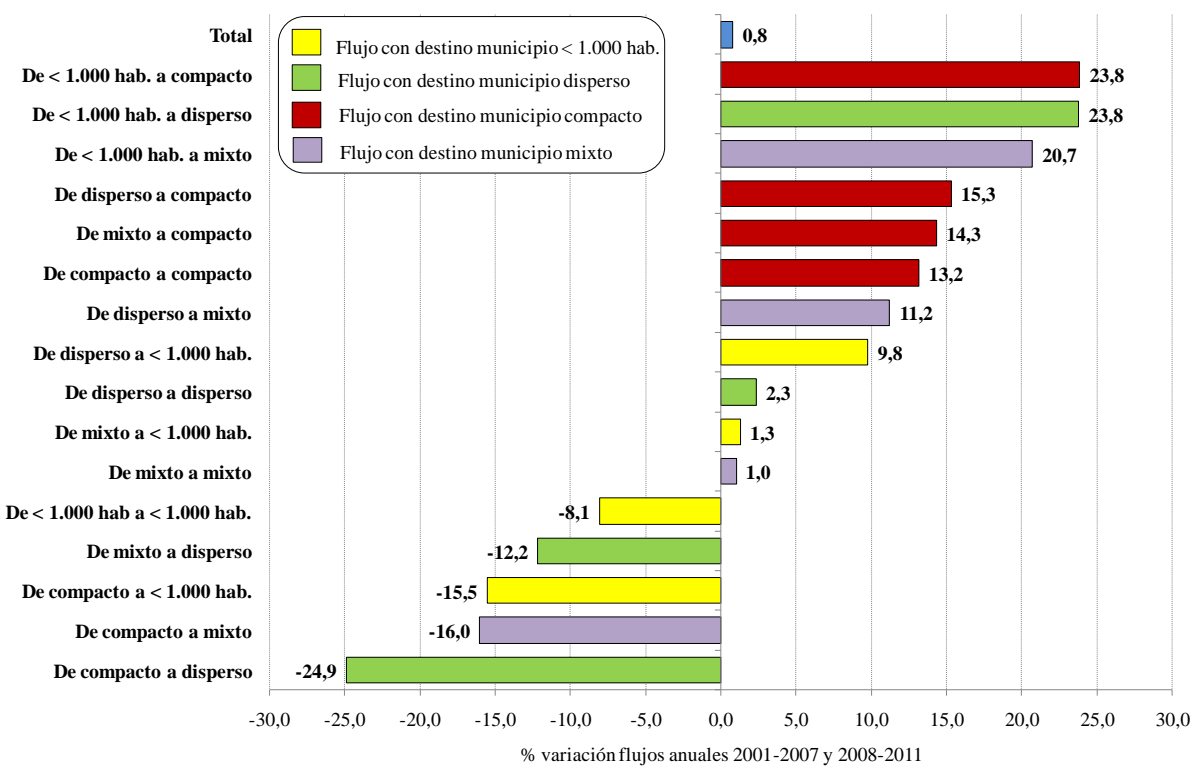

Fuente: Elaboración propia a partir de INE, Estadística de Variaciones Residenciales. Fichero de Microdatos, 1996-2011.

Un mayor detalle de las interacciones migratorias aparece en el cuadro 6 y en la figura 13. Se han tomado en cuenta cuatro situaciones de origen y destino: menor de 1.000 habitantes, compacto (suma de los dos grupos), mixto y disperso (suma de los dos grupos) y se ha comparado el promedio anual de los flujos migratorios de los dos periodos más significativos, el de 2001-07 y el de 2008-2011. En primer lugar 
conviene destacar como los promedios anuales de ambos períodos son muy semejantes lo que nos llevaría a considerar, engañosamente, que la crisis no ha tenido una repercusión notoria en la movilidad residencial. Ya hemos visto con detalle anteriormente el descenso significativo de las tasas migratorias en los últimos años.

Es evidente que las migraciones de los menores de 1.000 habitantes son las de menor volumen dada su menor población. Como lugar de destino, su atracción ha retrocedido para la población procedente de los municipios compactos en un 15,5\%. En cambio, ha pasado a ser el destino de una mayor proporción para los residentes en municipios dispersos, su incremento ha sido de un $9,8 \%$. No disponemos de mayor información pero sería plausible considerar que son áreas de expansión residencial de los municipios dispersos, que han agotado en parte su capacidad de atracción.

Por su parte, las migraciones con origen y/o destino a los municipios compactos son cuantitativamente las de mayor dimensión, especialmente la migración entre municipios compactos que llega a cifras anuales superiores a los 50.000 migrantes, representando casi el $40 \%$ del total de la movilidad intrametropolitana. El destino hacia estos municipios ha aumentado desde todo tipo de procedencias: desde los < 1.000 habitantes, los mixtos, los dispersos y también desde los propios compactos. La crisis económica está dando lugar a una disminución de la dispersión residencial hacia municipios de baja densidad.

Las interacciones migratorias con los municipios mixtos presentan una diversidad de situaciones. Por un lado, un descenso importante, del 16\%, de la migración de los municipios compactos hacia los mixtos y, de otro lado, un incremento de la migración de los municipios dispersos y de los $<1.000$ habitantes hacia los mixtos. Todo apunta hacia un menor grado de dispersión y de mayor concentración de la movilidad residencial.

Por último, los intercambios migratorios con los municipios dispersos son los que más han retrocedido. El descenso de las salidas desde los compactos a los dispersos ha disminuido en un $25 \%$ y la de los mixtos a los dispersos en un $12 \%$. Sólo consigue mantener su atracción con los municipios $<1.000$ habitantes y con ellos mismos. De nuevo se manifiesta la menor atracción de los municipios dispersos en estos últimos años.

Las opciones residenciales que más han aumentado están relacionadas con los municipios compactos y mixtos. Sólo excepcionalmente aumenta el intercambio entre los dispersos y los $<1.000$ habitantes en ambas direcciones. Y a la inversa, las relaciones migratorias que más han disminuido están relacionadas con el destino hacia los dispersos y los $<1.000$ habitantes seguidos en menor intensidad por los mixtos.

\section{Conclusión y discusión}

La movilidad residencial de la RMB desde la década de los 90 hasta 2007 se ha regido por una serie de pautas, tales como unas altas tasas de migración intrametropolitana, una proporción creciente de extranjeros -que casi llegan a representar la mitad de las migraciones internas en el ámbito de la Región-, así como una relación inversa entre 
intensidad migratoria y grado de compacidad de los municipios. En este sentido, existía un rol claramente diferenciado entre municipios dispersos y compactos, con tasas muy intensas tanto de crecimiento como de migración neta para los primeros e indicadores mucho más modestos para los segundos, que registraban valores de signo negativo en el caso de los de mayor densidad.

La pregunta central de este artículo abordaba el efecto de la crisis en la movilidad intrametropolitana de la RMB, tomando este patrón de comportamiento como punto de referencia inicial. Se partía de la premisa de que un cambio de coyuntura económica de las dimensiones del vivido a partir de 2008 tendría un efecto en las tendencias migratorias, modificando los rasgos presentados en la etapa inmediatamente anterior. Sin embargo, un primer análisis de los datos más recientes (2008-2011) mostraba un débil descenso de la movilidad residencial de la RMB cuando se examina de forma global, proporcionando una impresión engañosa de que la movilidad residencial no se estaba viendo alterada como consecuencia de la crisis, al menos no de forma drástica. El posterior análisis realizado, olvidando los datos agregados y adoptando una perspectiva detallada, ha hecho aflorar valoraciones distintas o, lo que es lo mismo, el verdadero impacto de la crisis.

La crisis, tal y como se ha demostrado, tiene un efecto desigual según colectivos y territorios. En primer lugar, la reducción de la movilidad es mucho más severa en la población extranjera que en la española, coincidiendo con los estudios que subrayan la mayor vulnerabilidad de este colectivo en esta etapa de crisis. No obstante, su movilidad sigue triplicando la de los españoles, demostrando que el cambio de domicilio es una estrategia incorporada al proceso de asentamiento de los extranjeros residentes en España. En segundo lugar, el grupo de 25-34 años y los mayores de 55 años -con excepción del grupo de 85 y más años- son los que notan un freno más acentuado de su movilidad. Se trata de dos momentos clave en el ciclo de vida migratorio: la edad de emancipación y formación de hogar propio -muy tardía en el caso español- y las edades próximas a la jubilación. En el pasado, estos dos momentos alimentaban las migraciones intrametropolitanas y, como consecuencia de la crisis, reducen su intensidad. Las únicas edades que aumentan su movilidad entre estos dos momentos son el grupo de 15 a 24 años. Este segmento de edad se ve afectado por un fuerte cambio en su composición entre 2001-2007 y 2008-2011 debido a la creciente participación de los extranjeros. Cabe recordar que, en la etapa más reciente, los extranjeros son casi la mitad de los que se desplazan a estas edades. En lo que se refiere a los mayores de 85 años, el aumento de su movilidad puede relacionarse con movimiento de arrastre, siguiendo los cambios de residencia experimentados previamente por sus hijos o bien por un incremento de la institucionalización de los mismos, con una amplia oferta ubicada también en los municipios dispersos, aprovechando su potencialidad para albergar residencias para mayores.

En tercer lugar, son los municipios dispersos los que, con diferencia, disminuyen su capacidad de atracción migratoria de forma más aguda, fruto de una drástica reducción de los cambios residenciales que los eligen como destino. En cambio, los municipios de mayor densidad experimentan una trayectoria totalmente opuesta, viendo crecer ahora su atractivo en el proceso de selección residencial.

El sensible descenso de las migraciones intrametropolitanas hacia zonas urbanas de baja densidad durante el último período parece lógico si se recuerda el peso interno de 
los motivos relacionados con las características de la vivienda y de su entorno. La restricción del acceso al crédito para la compra de un inmueble, el coste superior de la vida en disperso o la mejora de la oferta residencial en los municipios de mayor compacidad -con una ampliación y diversificación de la oferta y un ajuste de los precios medios tanto de compra como de alquiler- son algunos de los aspectos que explican la pérdida de atracción de los municipios dispersos. Sin embargo, esta situación no es óbice para que los municipios de alta dispersión sean, todavía, los que presentan tasas de migración neta más favorables en el conjunto de la RMB y unas tasas de inmigración intrametropolitanas destacadas. En este sentido, todo apunta hacia la pervivencia de este tipo de flujos, si bien ahora con una dimensión mucho más restringida. Así, familias que no notan el efecto de la crisis en su economía, que siguen valorando la calidad de vida del disperso, que ya disponen de una vivienda en dichas áreas o que asumen el coste diferencial de la misma siguen inclinándose por esta opción residencial. Sin embargo, los valores residenciales de las ciudades densas (la mayor proximidad a todo tipo de equipamientos y servicios o a los grandes mercados laborales y su mejor conexión por medio de transporte público y privado) recuperan atractivo en los momentos de crisis.

A pesar de la falta de estadísticas que permitan aquilatar su peso, las tendencias constatadas parecen indicar que la crisis ha cambiado las estrategias residenciales de muchas familias, al igual que ha trastocado sus ingresos. Por un lado, se deduce la disminución de aquellas que realizaban una migración para vivir en un contexto urbano de baja densidad con la única finalidad de una mejora residencial vinculada a la calidad medioambiental y de la vivienda. Todo apunta a que las migraciones que responden a estos objetivos son canceladas o aplazadas temporalmente, como se deduce del severo freno de los flujos desde el compacto hacia el disperso. Sin embargo, este hecho no debería interpretarse como una pérdida de relevancia de la vivienda en la movilidad residencial, si bien sí se apreciaría un cambio sensible respecto a la etapa anterior. Ahora, los motivos emergentes estarían relacionados con un menor coste de la misma, la aproximación a un mercado de alquiler que ofrezca precios más competitivos, el reparto entre padres e hijos de la residencia principal y la secundaria o incluso un traslado definitivo de las familias que tienen dos inmuebles con el fin de vender o alquilar la mejor posicionada en el mercado actual y mudarse hacia la peor valorada por el mismo. El acercamiento al lugar de trabajo con el fin de disminuir costes en transporte -aunque no se haya cambiado de trabajo-, la búsqueda de una vivienda de menor tamaño -con un coste inferior en mantenimiento, en impuestos...- o, simplemente, traslados que permitan un nuevo contrato de alquiler renegociado a la baja serían situaciones que estarían en alza en un momento de crisis como el actual y con un mercado de la vivienda con un fuerte excedente. El resultado es un mantenimiento de la intensidad de la movilidad pero con una composición distinta y, sobre todo, con una direccionalidad renovada. En este sentido, no parece que las motivaciones residenciales vayan a la baja, tal y como pronostican García Docampo y Otero ${ }^{35}$, sino que éstas están adquiriendo un nuevo contenido. En este sentido, surge la duda de cómo se clasifican este tipo de motivos muy atentos a los costes asociados al domicilio de residencia, es decir, si se categorizan como residenciales o como económicos.

\footnotetext{
${ }^{35}$ García Docampo y Otero, 2012.
} 
Mirando al pasado y al presente, la imagen que se obtiene es que nos encontramos en un momento de transición cuyo final está todavía por escribir. La etapa 2008-2011 mantiene, todavía, el papel jugado anteriormente por las distintas categorías según grado de compacidad. No obstante, tanto municipios dispersos como compactos manifiestan tendencias inequívocas de cambio. De momento, la fuerza de estos cambios no ha conseguido transformar las inercias anteriores y únicamente ha suavizado el rol desempeñado anteriormente. Sin embargo, en caso de continuar en el mismo sentido, su participación en la movilidad intrametropolitana de la RMB se modificaría. En este sentido, más que un agotamiento de un modelo, los cambios parecen ser una respuesta adaptativa a la nueva situación de nuestro país.

En definitiva, la riqueza de los intercambios migratorios intrametropolitanos de la actualidad subraya la complejidad del momento actual, también a nivel residencial, y obliga a mantener el interés en su trayectoria futura.

\section{Bibliografía}

AJA, E., ARANGO, J. y OLIVER, J. Anuario de Inmigración en España. 2012. Barcelona: CIDOB, 2013

AJA, E., ARANGO, J. y OLIVER, J. La inmigración en tiempos de crisis. Barcelona: CIDOB, 2009.

ALABART, A. Mobilitat residencial, solidaritat familiar i ciutadania en les regions metropolitanes. Revista Catalana de Sociologia, 2007, nº 22, p. 12-13.

ALABART, A. y LÓPEZ-VILLANUEVA, C. Familias, hogares y viviendas en las regiones metropolitanas. El caso de Barcelona. Cadernos Metrópole, 2007, vol. 17, p. 81-102.

BAYONA, J. y GIL-ALONSO, F. Suburbanisation and international immigration: The case of The Barcelona Metropolitan Region (1998-2009). Tijdschriftvoor economische en sociale geografie, 2012, doi:10.1111/j.1467-9663.2011.00687.x., Vol. 103 (3), p. 312-329.

BAYONA, J., GIL-ALONSO, F y PUJADAS, I. La evolución demográfica de las áreas metropolitanas españolas: entre el boom migratorio y la crisis económica e Inmobiliaria. En COMITÉ ESPAÑOL DE LA UGI (ed.) Aportación española al XXXII Congreso de la UGI, p. 146-161, 2012.

BAYONA, J. y LÓPEZ-GAY, A. Concentración, segregación y movilidad residencial de los extranjeros en Barcelona. Documents d'Anàlisi Geogràfica, 2011, n 57, p. 381412.

BENDIXSON, T. Push-Pull Forces in the Spatial Organization of Greater London and South East England. En RICHARDSON, H.W. y BAE, C.H.C. (eds.), Urban Sprawl in Western Europe and the United States. London: Ashagate, 2004. 
BOURNE, L.S. Reurbanization, uneven urban development and the debate on new urban forms. Urban Geography, 1996, vol.17, nº 8, p. 690-713.

BRANDIS, D. Los grandes desarrollos residenciales de la periferia de Madrid: de la burbuja a la crisis inmobiliaria. En Espacios y paisajes urbanos: reflexionar sobre su presente para proyectar su futuro. Santander: AGE, Universidad de Oviedo, Universidad de Cantabria y Universidad del País Vasco, 2010.

CHAMPION, A. Urbanization, Suburbanization, Counterurbanization and Reurbanization. In PADDISON, R. (ed.) Handbook of Urban Studies. London: SAGE, p. 143-161, 2001.

CHESIRE, P.C. A new phase of urban development in Eastern Europe?. The evidence for the 1980's. Urban Studies, 1995, vol. 32, nº 7, p. 1045-1063.

CHESHIRE, P.C. Resurgent cities, urban myths and policy hubris: what we need to know. Urban Studies, 2006, nº 43, p. 1231-1246.

COLECTIVO IOÉ. Impactos de la crisis sobre la población inmigrante. Colectivo Ioé-Organización, 2012.

DOMINGO, A. y RECAÑO, J. La inflexión en el ciclo migratorio internacional en España: impacto y consecuencias demográficas. En AJA, E. et al. La inmigración en tiempos de crisis. Barcelona: CIDOB, 2009, p. 182-209.

DOMÍNGUEZ MUJICA, J. La huella de la crisis económica en la demografía y sociedad españolas: más interrogantes que certezas. En LÓPEZ PALOMEQUE, F. et al. Barcelona: Universitat de Barcelona, en prensa.

DOMÍNGUEZ MUJICA, J., GUERRA, R. y PARREÑO, J. Migration at time of Global Economic Crisis: The situation in Spain. International Migration, 2012, doi: 10.1111/inmig.12023, vol. 52, no 6, p. 113-127.

FIELDING, T. Counterurbanization in Western Europe. Progress and Planning, 1982, vol. 17, no 1-2, p. 1-52.

GARCÍA-ALMIRALL, P., FULLAONDO, A. y FRIZZERA, A. Inmigración y espacio socio-residencial en la Región Metropolitana de Barcelona. Ciudad y Territorio. Estudios Territoriales, 2008, nº 158, 727-744

GARCÍA COLL, A. Migraciones interiores y transformaciones territoriales. Papeles de Economía Española, 2005, n 104, 76-91.

GARCÍA COLL, A. Migraciones interiores y transformaciones territoriales. En PONS, J.J. et al.: Territorio y movilidad interior de la población en España. Pamplona: Ediciones Universidad de Navarra, S.A. (EUNSA), p. 13-40

GARCÍA COLL, A. The process of residential sprawl in Spain: Is it really a problem?. Urban Research \& Practice, 2011, vol. 4, nº 3, p. 250-263. 
GARCÍA DOCAMPO, M. y OTERO, R. Transición territorial: modelo teórico y contraste con el caso español. Reis. Revista Española de Investigaciones Sociológicas, 2012, 139, p. 133-161.

GIL-ALONSO, F., BAYONA, J. y PUJADAS, I. From boom to crash: Spanish urban areas in a decade of change (2001-2011). European Urban and Regional Studies. 2016, doi:10.1177/0969776413498762, n. 2, p. 198-216.

GIL-ALONSO, F., BAYONA, J. y PUJADAS, I. ¿Es la crisis un freno a la suburbanización?: su impacto sobre la movilidad residencial de las principales áreas metropolitanas de España”. En GOZÁLVEZ, V. y MARCO, J.A. (Ed.) Urbanismo expansivo: de la utopía a la realidad, Actas del XXII Congreso de Geógrafos españoles, Alicante: AGE-Universidad de Alicante, p. 293-305, 2011.

GREENWOOD, M.J Internal migration in developed countries. In ROSENZWEIG, M. R. y STARK, O. (eds.). Handbook of Population and Family Economics, Elsevier, vol.1, p. 647-720, 1993.

HENRY, G. Análisis de costes de la baja densidad. Una lectura desde la sostenibilidad. En INDOVINA, F. (coord.). La ciudad de baja densidad: lógicas, gestión y contención. Barcelona: Diputación de Barcelona, 2007, p. 203-242

IERMB. Enquesta de condicions de vida i hàbits de la població de Catalunya, 2011. Resultats sintètics: Barcelona: ciutat, àrea metropolitana, Regió metropolitana, província. Barcelona: IERMB, AMB y Diputació de Barcelona, 2012.

KABISCH, N. y HAASE, D. Diversifying European Agglomerations: Evidence of Urban Population Trends for the 21st Century. Population, Space and Place, 2011, n. 17, p. 236-253.

LEAL, J. El diferente modelo residencial de los países del sur de Europa: el mercado de viviendas, la familia y el Estado. Arxius, 2004, 10, p.11-37.

LÓPEZ COLÁS, J., MÓDENES, J.A. y YÉPEZ, B. Los usuarios de residencias secundarias en España: perfiles regionales. Boletín de la AGE, 2007, 45, p. 307-325.

LÓPEZ DE LUCIO, R. La incipiente configuración de una región urbana dispersa: el caso de la Comunidad Autónoma de Madrid (1960-1993). En MONCLÚS, F. (ed). La ciudad dispersa, Barcelona: Centre de Cultura Contemporània, 1998, p. 5-16.

LÓPEZ-GAY, A. ¿Vuelve el centro? Caracterización demográfica de los procesos de reurbanización en las metrópolis españolas. En PUJADAS, I. et al. (eds). Población y espacios urbanos. Actas del XII Congreso de la Población española, Barcelona, p. 163-180, 2011.

LÓPEZ-VILLANUEVA, C. y ALABART, A. Crisis económicas, viviendas y estrategias familiares. Comunicación Congreso Español Sociología. Julio 2010. Madrid. 
MIRET, P. Vivir aún en casa o tener casa propia: emancipación residencial en España, 1976-2007. ACE, Arquitectura, Ciudad y Territorio, 2007, vol. 2, nº 5, p. 301-329.

MUÑIZ, I., GARCÍA, M.A y CALATAYUD, D. Sprawl. Definición, causas y efectos. Working Paper del Departament d'Economia Aplicada 06.03, 2006, Bellaterra: Universitat Autònoma de Barcelona.

MUÑOZ, F. Urbanalización. Paisajes comunes, lugares globales. Barcelona: Gustavo Gili, 2010.

MUÑOZ, F. Coord. Estratègies vers la ciutat de baixa densitat: De la contenció a la gestió, Barcelona: Diputació de Barcelona, 2011.

NEL $L$ LO, O. La tercera fase del proceso de metropolización en España. In AGE (ed.). Los procesos urbanos postfordistas. Palma de Mallorca: Universitat de les Illes Balears y Asociación de Geógrafos Españoles, 2007, p. 19-33.

POZO, E. y GARCÍA PALOMARES, J.C. Inmigración y cambio demográfico en la región metropolitana madrileña entre 1996 y 2006, Anales de Geografía de la Universidad Complutense, 2009, vol. 29, n 1, p. 111- 138.

POZO E. y RODRÍGUEZ, J. Transformaciones sociodemográficas recientes en las comarcas Castellano Manchegas limítrofes con la Comunidad de Madrid. Anales de Geografía, 2006, 26, p. 249-281.

PUJADAS, I. PRATS, P. y COLL, M. Elección residencial y nuevas formes urbanas. El caso de la Región Metropolitana de Barcelona. En Los procesos urbanos postfordista, Grupo de Geografía Urbana de la Asociación de Geógrafos Españoles, Palma de Mallorca, Universitat de les Illes Balears y Asociación de Geógrafos Españoles, 2007, p. 215-234.

PUJADAS, I. Movilidad residencial y expansión urbana en la Región Metropolitana de Barcelona, 1982-2005. Scripta Nova. Revista Electrónica de Geografía y Ciencias Sociales, 2009, vol. XIII, 290.

PUJADAS, I. PRATS, P. y COLL, M.: Migrar de la ciudad: la elección residencial en la Región Metropolitana de Barcelona. En J. PONS et al. (eds.), Territorio y movilidad interior de la población en España, Pamplona, EUNSA, 2009, p.137-147.

PUJADAS, I. y BAYONA, J. Las migraciones residenciales en las regiones metropolitanas de Barcelona y Madrid. En DOMÍNGUEZ, M. y LÓPEZ, C. (Coords.) Barcelona y Madrid: Procesos urbanos y dinámicas sociales. Editorial Síntesis, 2015, pp. 43-70. ISBN: 978-84-907722-5-6.

PUMARES, P, GARCÍA-COLL, A. y ASENSIO, M.A. La movilidad laboral y geográfica de la población extranjera en España. Madrid: Ministerio de Trabajo y Asuntos Sociales, 2006. 
REHER, D. (ed). Informe Encuesta Nacional de Inmigrantes. (ENI-2007). Madrid: INE, 2008.

REHER, D., REQUENA, M. y SANZ, A. ¿España en la encrucijada?. Consideraciones sobre el cambio de ciclo migratorio. Revista Internacional de Sociología, 2011, Monográfico, 1, p. 9-44.

RICHARDSON, H.W. y BAE, C.H.C. (eds) Urban Sprawl in Western Europe and the United States. London: Ashagate, 2004.

ROCA, J., ARELLANO, B. y MOIX, M. Estructura urbana, policentrismo y sprawl: los ejemplos de Madrid y Barcelona. Ciudad y territorio, 2011, 168, p. 299-321.

RÓDENAS, C. Emigración y economía en España, Alicante: Universidad de Alicante y Editorial Civitas. 1994.

RODRÍGUEZ LÓPEZ, J. El mercado de la vivienda tras la recaída de la crisis. Ciudad y territorio: Estudios territoriales, 169-170, 2011, p. 701-716.

ROGERS, A. Requiem for the Net Migrant. Geographical Analysis, 22, p. 283-300. doi: 10.1111/j.1538-4632.1990.tb00212.x, 1990.

RUÍZ ALMAR, E. Las grandes aglomeraciones urbanas en Europa y en España, En FERIA, J.M ${ }^{a}$ y ALBERTOS, J.M. (Coords) La ciudad metropolitana en España. Procesos urbanos en los inicios del siglo XX. Navarra: Civitas-Thomson Reuters, 2010, p. 125-148.

SUSINO, J. y DUQUE, R. Veinte años de suburbanización en España (1981-2001). El perfil de sus protagonistas. Documents d'Anàlisi Geogràfica, 2013, 59, 2, p.265-290

VALENZUELA, M. y SALOM, J. Los procesos de urbanización en España. Nuevos factores, nuevas tendencias. En CÁNOVAS, G. et al (eds) España y el mediterráneo: una reflexión desde la geografía española: aportación española al XXXI Congreso de la Unión Geográfica Internacional. Madrid: Comité Español de la Unión Geográfica Internacional, 2008, p. 49-56.

VAN OURS, J.C. y VEENMAN, J. The Netherlands: Old Emigrants-Youn Immigrant Country. En ZIMMERNANN, K.F. European migration: what do we know. Oxford: Oxford University Press, 2005, p. 173-196.

WARNES, T. Migration and life course. En STILLWELL, J. et al. (eds). Migration processes and patterns. Population redistribution in the United Kingdom. London: Belhaven Press, 1992, p. 175-187.

C Copyright Arlinda García Coll,2016

(c) Copyright Cristina López Villanueva, 2016

(c) Copyright Isabel Pujadas Rúbies, 2016.

(c) Copyright Scripta Nova, 2016. 
Ficha bibliográfica:

GARCÍA COLL, Arlinda; LÓPEZ, Cristina; PUJADAS, Isabel. Movilidad residencial en tiempos de crisis. El caso de la Región Metropolitana de Barcelona. Scripta Nova. Revista Electrónica de Geografía y Ciencias Sociales. [En línea]. Barcelona: Universidad de Barcelona, 30 de noviembre de 2016, vol. XX, nº 549-4. <http://www.ub.es/geocrit/sn/sn-5494.pdf>. ISSN: 1138-9788. 